\title{
SOME DISTRIBUTIONAL RESULTS FOR POISSON-VORONOI TESSELLATIONS
}

\author{
VOLKER BAUMSTARK * AND \\ GÜNTER LAST, ${ }^{* * *}$ Universität Karlsruhe
}

\begin{abstract}
We consider the Voronoi tessellation based on a stationary Poisson process $N$ in $\mathbb{R}^{d}$. We provide a complete and explicit description of the Palm distribution describing $N$ as seen from a randomly chosen (typical) point on a $k$-face of the tessellation. In particular, we compute the joint distribution of the $d-k+1$ neighbours of the $k$-face containing the typical point. Using this result as well as a fundamental general relationship between Palm probabilities, we then derive some properties of the typical $k$-face and its neighbours. Generalizing recent results of Muche (2005), we finally provide the joint distribution of the typical edge (typical 1-face) and its neighbours.
\end{abstract}

Keywords: Voronoi tessellation; Poisson process; random measure; Palm distribution; typical face; typical edge

2000 Mathematics Subject Classification: Primary 60D05; 60G55

\section{Introduction}

Let $N$ be a stationary Poisson process on $\mathbb{R}^{d}$ with finite intensity $\lambda>0$. The Voronoi cell, $C(x)$, of $x \in N$ is the set of all points $y \in \mathbb{R}^{d}$ whose distances from $x$ are smaller than or equal to their distances from all other points of $N$. The subject of this paper is the PoissonVoronoi tessellation $\{C(x): x \in N\}$. Voronoi tessellations can be defined for more general point processes than $N$. They are fundamental models in stochastic geometry and constitute arguably one of the most popular types of mathematical model in applications; see [7], [10], [11], and the references therein. We will present a complete and explicit description of the Palm probability measure $\mathrm{P}_{k}^{0}, k \in\{0, \ldots, d\}$, which describes the statistical behaviour of $N$ as seen from a typical point chosen 'uniformly' on the $k$-faces of the tessellation. In particular, we obtain the joint distribution of the $d-k+1$ neighbours of the $k$-dimensional face containing this typical point. For $k=0$, our result essentially boils down to the distribution of the typical cell of the Poisson Delaunay tessellation, established in [5] and [6]. In fact, our present approach owes much to these seminal papers.

To formulate our main result we introduce some notation. We are working in $\mathbb{R}^{d}$ with Euclidean norm $|\cdot|$, scalar product $\langle\cdot, \cdot\rangle$, and unit sphere $S^{d-1}:=\left\{z \in \mathbb{R}^{d}:|z|=1\right\}$. The $i$-dimensional Hausdorff measure is denoted by $\mathscr{H}^{i}$. The closed ball with centre $a \in \mathbb{R}^{d}$ and radius $r \geq 0$ is denoted by $B(a, r)$, while $B^{0}(a, r)$ denotes the corresponding open ball. The volume $\overline{\mathcal{H}}^{d}(B(0,1))$ of the unit ball in $\mathbb{R}^{d}$ is denoted by $\kappa_{d}$. For $k \in\{0, \ldots, d\}$, let $s_{k}$ be the system of all $k$-faces of the Voronoi cells $C(x), x \in N$ (see Subsection 2.2 for related notation).

Received 24 May 2006; revision received 29 November 2006.

* Postal address: Institut für Stochastik, Universität Karlsruhe (TH), 76128 Karlsruhe, Germany.

** Email address: last@math.uni-karlsruhe.de 
We define the following stationary random measure on $\mathbb{R}^{d}$ :

$$
M_{k}:=\sum_{F \in \oiint_{k}} \mathscr{H}^{k}(F \cap \cdot) .
$$

It is well known [5], [6], [10] that the intensity, $\mu_{k}:=\mathrm{E}\left[M_{k}\left([0,1]^{d}\right)\right]$, of $M_{k}$ is given by

$$
\begin{aligned}
\mu_{k}= & \lambda^{(d-k) / d} \frac{2^{d-k+1} \pi^{(d-k) / 2}}{d(d-k+1) !} \\
& \times \frac{\Gamma(d-k+k / d)}{\Gamma((k+1) / 2)} \frac{\Gamma\left(\left(d^{2}-d k+k+1\right) / 2\right) \Gamma(1+d / 2)^{d-k+k / d}}{\Gamma\left(\left(d^{2}-d k+k\right) / 2\right) \Gamma((d+1) / 2)^{d-k}} .
\end{aligned}
$$

Consider the Palm probability measure, $\mathrm{P}_{k}^{0} \equiv \mathrm{P}_{M_{k}}^{0}$, of $M_{k}$ (defined in Subsection 2.1). In the (rather trivial) case in which $k=d, \mathrm{P}_{k}^{0}$ equals the underlying (stationary) probability measure. Under $\mathrm{P}_{k}^{0}$ the origin $0 \in \mathbb{R}^{d}$ can be interpreted as a typical point of $M_{k}$. Almost surely with respect to $\mathrm{P}_{k}^{0}$, there are exactly $d-k+1$ different points, $X_{k, 0}, \ldots, X_{k, d-k} \in N$, such that

$$
R_{k}:=\left|X_{k, 0}\right|=\cdots=\left|X_{k, d-k}\right|
$$

and

$$
N \cap B^{0}\left(0, R_{k}\right)=\varnothing .
$$

These are the neighbours of the $k$-face containing 0 . We may assume that $X_{k, 0}, \ldots, X_{k, d-k}$ are in general position, i.e. not contained in some affine space of dimension $d-k-1$. Hence, there exists a unique $(d-k)$-dimensional ball in the affine hull of these points containing the points on its boundary. We let $Z_{k}$ denote the centre of this ball. The case in which $d=3$ and $k=1$ is illustrated in Figure 1. For $k \leq d-1$, the random variable

$$
R_{k}^{\prime}:=\left|X_{k, 0}-Z_{k}\right|
$$

is $\mathrm{P}_{k}^{0}$-almost surely positive and we define

$$
U_{k, 0}:=\frac{X_{k, 0}-Z_{k}}{R_{k}^{\prime}}, \quad \ldots, \quad U_{k, d-k}:=\frac{X_{k, d-k}-Z_{k}}{R_{k}^{\prime}} .
$$

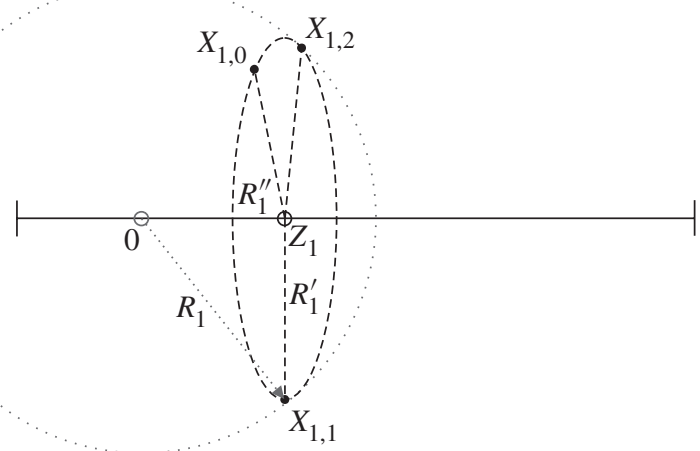

FIgure 1: The situation under $\mathrm{P}_{1}^{0}$, for $d=3$. 
For $k=d$, we $\mathrm{P}_{k}^{0}$-almost surely have $X_{k, 0}=Z_{k}$ and we define $R_{k}^{\prime}:=0$ and $U_{k, 0}:=0$. Furthermore, for $k \geq 1$ the random variable

$$
R_{k}^{\prime \prime}:=\left|Z_{k}\right|
$$

is $\mathrm{P}_{k}^{0}$-almost surely positive and we define

$$
U_{k}:=\frac{Z_{k}}{R_{k}^{\prime \prime}} .
$$

For $k=0$, we have $Z_{k}=0$ and we define $R_{k}^{\prime \prime}:=0$ and $U_{k}:=0$. From $\left|X_{k, 0}\right|=\cdots=\left|X_{k, d-k}\right|$ it easily follows that $U_{k}$ is orthogonal to the affine hull of $U_{k, 0}, \ldots, U_{k, d-k}$. In particular,

$$
R_{k}^{2}=R_{k}^{\prime 2}+R_{k}^{\prime \prime 2}
$$

Under the Palm probability measure $\mathrm{P}_{k}^{0}$, the random variables $R_{k}^{\prime}, R_{k}^{\prime \prime}, U_{k, 0}, \ldots, U_{k, d-k}$, and $U_{k}$ provide a natural description of the neighbours of the $k$-face containing 0 :

$$
N \cap B\left(0, R_{k}\right)=\left\{R_{k}^{\prime \prime} U_{k}+R_{k}^{\prime} U_{k, i}: i=0, \ldots, d-k\right\} \quad \mathrm{P}_{k}^{0} \text {-almost surely. }
$$

It is convenient to write

$$
\Psi_{k}:=\left\{U_{k, 0}, \ldots, U_{k, d-k}\right\} .
$$

For $k \leq d-1$, this is a finite point process on the unit sphere $S^{d-1}$.

The following theorem gives a complete and explicit description of the Palm probability measure, $\mathrm{P}_{k}^{0}$, of $M_{k}$. We fix a $(d-k)$-dimensional linear subspace $L \subset \mathbb{R}^{d}$ and denote by $\mathbb{S}_{L}$ and $\mathbb{S}_{L^{\perp}}$ the uniform distributions on the unit spheres in $L$ and, respectively, in the orthogonal complement, $L^{\perp}$, of $L$. In the degenerate case, in which $L=\{0\}$, we let $\mathbb{S}_{L}$ be the Dirac measure at 0 .

Theorem 1.1. Assume that $N$ is a stationary Poisson process of intensity $\lambda>0$ and let $k \in\{0, \ldots, d\}$. Then the following assertions hold under $\mathrm{P}_{k}^{0}$.

(i) The random variables $\left(\left\{x \in N:|x|>R_{k}\right\}, R_{k}\right), R_{k}^{\prime 2} / R_{k}^{2}$, and $\left(\Psi_{k}, U_{k}\right)$ are independent.

(ii) $R_{k}^{d}$ is gamma distributed with shape parameter $d-k+k / d$ and scale parameter $\lambda \kappa_{d}$.

(iii) The conditional distribution of $\left\{x \in N:|x|>R_{k}\right\}$ given $R_{k}=r$ can be chosen to be the distribution of a homogeneous Poisson process on the complement of the ball $B(0, r)$, with intensity $\lambda$.

(iv) For $k \in\{1, \ldots, d-1\}, R_{k}^{\prime 2} / R_{k}^{2}$ has a beta distribution with parameters $d(d-k) / 2$ and $k / 2$.

(v) The random pair $\left(\Psi_{k}, U_{k}\right)$ has distribution

$$
\begin{aligned}
\mathbb{Q}_{k}:=c_{k}^{-1} \int \cdots \int & 1_{\left\{\left(\left\{\vartheta u_{0}, \ldots, \vartheta u_{d-k}\right\}, \vartheta u\right) \in \cdot\right\}} \Delta_{d-k}\left(u_{0}, \ldots, u_{d-k}\right)^{k+1} \\
& \times \mathbb{S}_{L}\left(\mathrm{~d} u_{0}\right) \cdots \mathbb{S}_{L}\left(\mathrm{~d} u_{d-k}\right) \mathbb{S}_{L^{\perp}}(\mathrm{d} u) v(\mathrm{~d} \vartheta)
\end{aligned}
$$


where $c_{d}:=1, c_{d-1}:=2^{d-1}$, and, for $k<d-1$,

$$
\begin{aligned}
c_{k}:= & \frac{1}{((d-k) !)^{k+1}}\left[\frac{\Gamma((d-k) / 2)}{\Gamma((d+1) / 2)}\right]^{d-k} \frac{\Gamma\left(\left(d^{2}-k d+k+1\right) / 2\right)}{\Gamma\left(\left(d^{2}-k d\right) / 2\right)} \\
& \times \frac{\Gamma((k+2) / 2) \cdots \Gamma(d / 2)}{\Gamma\left(\frac{1}{2}\right) \cdots \Gamma((d-k-1) / 2)} ;
\end{aligned}
$$

$\Delta_{d-k}\left(u_{0}, \ldots, u_{d-k}\right)$ is the $(d-k)$-dimensional volume of the simplex spanned by the vectors $u_{0}, \ldots, u_{d-k}$ (and is identically equal to 1 for $k=d$ ); and $v$ is the uniform distribution on the rotation group $\mathrm{SO}_{d}$.

The proof of this theorem is given in Section 3 and is a straightforward application of wellestablished methods (see [5], [6], [7], and [10]). Despite the lack of a novel method, it is pleasing that the whole proof is the result of just one calculation. In the special case in which $k=0$ (mentioned above), the integration with respect to $v$ can be dropped. For $k=d$ (i.e. $\mathrm{P}_{k}^{0}=\mathrm{P}$ ), the result is not only well known but also almost trivial.

Based on Theorem 1.1 and a general relationship between Palm probabilities (see Proposition 2.1), in Section 4 we will discuss some distributional results for the typical $k$-face. In particular, we derive the joint distribution of the directions to the neighbours of the typical $k$-face. In Section 5 we will then provide complete and explicit formulae for the joint distribution of the typical edge and its neighbours. In particular, we obtain the distributional results derived and cited in [8] in a more transparent and rigorous way.

\section{Poisson-Voronoi tessellations}

\subsection{Palm measures}

Let $N^{\prime}$ denote the set of all locally finite simple counting measures on $\mathbb{R}^{d}$, i.e. the set of all measures $\omega$ on $\mathbb{R}^{d}$ that are integer valued on bounded sets and have $\omega(\{x\}) \leq 1$ for all $x \in \mathbb{R}^{d}$. Any $\omega \in N^{\prime}$ is identified with its support $\left\{x \in \mathbb{R}^{d}: \omega(\{x\})>0\right\}$, a locally finite subset of $\mathbb{R}^{d}$ (i.e. by a point of $\omega$ we will mean a point in the support of $\omega$ ). Actually, $\omega$ is just the sum of the Dirac measures $\delta_{x}$ over all $x$ in the support of $\omega$. We let $\mathcal{N}^{\prime}$ denote the $\sigma$-field generated by the mappings $\omega \mapsto \omega(B), B \in \mathcal{B}^{d}$, where $\mathcal{B}^{d}$ denotes the Borel $\sigma$-field on $\mathbb{R}^{d}$. Following [7] we say that the points of $\omega \in N^{\prime}$ are in general quadratic position if both no $d+2$ points of $\omega$ lie on the boundary of some ball and any $k \in\{2, \ldots, d+1\}$ points $x_{1}, \ldots, x_{k} \in \omega$ are in general position, i.e. do not lie in a $(k-2)$-dimensional affine subspace of $\mathbb{R}^{d}$. We denote by $\boldsymbol{N}$ the measurable set of all $\omega \in N^{\prime}$ whose convex hull coincides with $\mathbb{R}^{d}$ and whose points are in general quadratic position. Note that $N$ is shift invariant, in the sense that $\omega \in N^{\prime}$ belongs to $N$ if and only if $\omega+x:=\{y+x: y \in \omega\} \in N$ for all $x \in \mathbb{R}^{d}$. We write $\mathcal{N}:=\mathcal{N}^{\prime} \cap \boldsymbol{N}$ and denote by $N$ the identity on $N$.

We consider a probability measure $\mathrm{P}$ on $(\boldsymbol{N}, \mathcal{N})$ such that $N$ is a homogeneous Poisson process of intensity $\lambda>0$. This is justified by the well-known fact (see [7] and [10]) that almost all realizations of a stationary Poisson process (with positive intensity) are in $N$. In this paper all random elements will be defined on the probability space $(\boldsymbol{N}, \mathcal{N}, \mathrm{P})$. Note that $\mathrm{P}$ is stationary in the sense that $\mathrm{P}(N+x \in \cdot)=\mathrm{P}$ for all $x \in \mathbb{R}^{d}$.

A random measure $M$ on $\mathbb{R}^{d}$ (see, e.g. [2]) is a random variable taking its values in the space, $\boldsymbol{M}$, of all locally bounded measures $\alpha$ on $\mathbb{R}^{d}$ equipped with the $\sigma$-field $\mathcal{M}$ generated by the mappings $\alpha \mapsto \alpha(B), B \in \mathscr{B}^{d}$. Note that $\boldsymbol{N}^{\prime}$ is a measurable subset of $\boldsymbol{M}$. A point process on $\mathbb{R}^{d}$ is a random measure $M$ satisfying $\mathrm{P}\left(M \in N^{\prime}\right)=1$. For a random measure $M$, 
it is convenient to write $M(\omega, B)$ instead of $M(\omega)(B)$. A random measure $M$ on $\mathbb{R}^{d}$ is said to be stationary if

$$
M(\omega, B+x)=M(\omega-x, B), \quad \omega \in N, x \in \mathbb{R}^{d}, B \in \mathcal{B}^{d} .
$$

If $M$ is a stationary random measure then the distribution of $M(\cdot+x)$ is the same for any $x \in \mathbb{R}^{d}$. The measure

$$
\mathrm{P}_{M}(A):=\iint 1_{\left\{\omega-x \in A: x \in[0,1]^{d}\right\}} M(\omega, \mathrm{d} x) \mathrm{P}(\mathrm{d} \omega), \quad A \in \mathcal{N},
$$

is called the Palm measure of $M$ (with respect to P); see [3]. This measure is $\sigma$-finite and satisfies the refined Campbell theorem, i.e.

$$
\mathrm{E}\left[\int f(N-x, x) M(\mathrm{~d} x)\right]=\mathrm{E}_{M}\left[\int f(N, x) \mathrm{d} x\right]
$$

for all measurable functions $f: N \times \mathbb{R}^{d} \rightarrow[0, \infty)$, where $\mathrm{E}_{M}$ denotes expectation with respect to $\mathrm{P}_{M}$ and $\mathrm{d} x$ indicates integration with respect to Lebesgue measure. If the intensity, $\lambda_{M}:=\mathrm{E}\left[M\left([0,1]^{d}\right)\right]$, of $M$ is positive and finite, then we can define the Palm probability measure, $\mathrm{P}_{M}^{0}:=\lambda_{M}^{-1} \mathrm{P}_{M}$, of $M$. The expectation of a random variable $\xi$ with respect to $\mathrm{P}_{M}^{0}$ is denoted by $\mathrm{E}_{M}^{0}[\xi]$.

\subsection{Voronoi tessellations}

Closely following [7] and Section 6.2 of [10], we now give a detailed description of Voronoi tessellations. We refer the reader with measurability questions to the latter monograph.

Let $\omega \in N$. The Voronoi cell, $C(\omega, x)$, of $x \in \omega$ consists of all points $y \in \mathbb{R}^{d}$ satisfying $|y-x| \leq \min \{|y-z|: z \in \omega\}$. By $\delta_{d}(\omega):=\{C(\omega, x): x \in \omega\}$ we then denote the Voronoi tessellation based on $\omega$. The elements of $\delta_{d}(\omega)$ are bounded and convex polytopes, and any bounded set is intersected by only finitely many cells in $\delta_{d}(\omega)$ (see Satz 6.2.1 of [10]). The subject of this paper is $\wp_{d}(N)$, the random tessellation based on the Poisson process $N$.

For $k \in\{0, \ldots, d-1\}$, a $k$-face (of $\delta_{d}(\omega)$ ) is a nonempty intersection of $d-k+1$ Voronoi cells (see also Satz 6.2 .3 of [10]). The system of all such $k$-faces is denoted by $\delta_{k}(\omega)$. A cell is referred to as a $d$-face. For $k \in\{0, \ldots, d\}$ and $x \in \mathbb{R}^{d}$, we write $F_{k}(\omega, x) \equiv F \in \delta_{k}(\omega)$ provided that $x$ is in the relative interior of $F$. For all other $x$, we define $F_{k}(x):=\{x\}$ and note the following covariance property:

$$
F_{k}(\omega, x)=F_{k}(\omega-x, 0)+x, \quad \omega \in N, x \in \mathbb{R}^{d} .
$$

The $k$-faces are generated in the following way. For points $x_{0}, \ldots, x_{d-k} \in \mathbb{R}^{d}$ in general position, we let $z\left(x_{0}, \ldots, x_{d-k}\right)$ denote the centre of the uniquely determined $(d-k)$-dimensional ball with $x_{0}, \ldots, x_{d-k}$ on its boundary. Furthermore, we let $F\left(x_{0}, \ldots, x_{d-k}\right)$ denote the $k$-dimensional affine space that is orthogonal to the above ball and contains $z\left(x_{0}, \ldots, x_{d-k}\right)$. Let $F \in \delta_{k}(\omega)$. Then there exist (lexicographically ordered) points $x_{0}, \ldots, x_{d-k} \in \omega$ such that

$$
F=\left\{x \in F\left(x_{0}, \ldots, x_{d-k}\right): B^{0}\left(x,\left|x-x_{0}\right|\right) \cap \omega=\varnothing\right\} .
$$


Conversely, given different points $x_{0}, \ldots, x_{d-k} \in \omega$ such that the thus-defined set $F$ has a nonempty relative interior, we have $F \in s_{k}(\omega)$.

Let $\omega \in N$, let $x$ be a point in the relative interior of some $F \in s_{k}(\omega)$, and choose $x_{0}, \ldots, x_{d-k} \in \omega$ as in (2.1). By definition of $N$ the set $\left\{x_{0}, \ldots, x_{d-k}\right\} \subset \omega$ is uniquely determined by (2.1), and we define

$$
\begin{gathered}
R_{k}(\omega, x):=\left|x-x_{0}\right|=\cdots=\left|x-x_{d-k}\right|, \\
X_{k, i}(\omega, x):=x_{i}, \quad i=0, \ldots, d-k, \\
Z_{k}(\omega, x):=z\left(x_{0}, \ldots, x_{d-k}\right) .
\end{gathered}
$$

For points $x \in \mathbb{R}^{d}$ that are not in the relative interior of some $k$-face, we set $R_{k}(\omega, x):=0$ and $X_{k, 0}(\omega, x)=\cdots=X_{k, d-k}(\omega, x)=Z_{k}(\omega, x):=x$. For $k=0$, we always have $Z_{k}(\omega, x)=x$. The mappings introduced above possess natural covariance and invariance properties with respect to shifts; e.g.

$$
\begin{gathered}
X_{k, i}(\omega, x)=X_{k, i}(\omega-x, 0)+x, \quad \omega \in N, x \in \mathbb{R}^{d}, \\
Z_{k}(\omega, x)=Z_{k}(\omega-x, 0)+x, \quad \omega \in N, x \in \mathbb{R}^{d}, \\
R_{k}(\omega, x)=R_{k}(\omega-x, 0), \quad \omega \in N, x \in \mathbb{R}^{d} .
\end{gathered}
$$

\subsection{Random measures associated with the Voronoi tessellation}

Recall from (1.1) that, for any $k \in\{0, \ldots, d\}$,

$$
M_{k}=\sum_{F \in \AA_{k}(N)} \mathscr{H}^{k}(F \cap \cdot)
$$

is a stationary random measure, with Palm probability measure $\mathrm{P}_{k}^{0} \equiv \mathrm{P}_{M_{k}}^{0}$.

We now construct another series of random measures using suitable centres of the $k$-faces. Let $k \in\{0, \ldots, d\}$ and denote by $\mathscr{P}_{k}$ the system of all $k$-dimensional, nonempty, bounded polytopes $F \subset \mathbb{R}^{d}$. We define a measurable mapping $\pi_{k}: N \times \mathcal{P}_{k} \rightarrow \mathbb{R}^{d}$ in the following way. For $\omega \in N$ and $F \in \delta_{k}(\omega)$, we choose an arbitrary point $y$ in the relative interior of $F$ and define $\pi_{k}(\omega, F):=Z_{k}(\omega, y)$. In all other cases, we define $\pi_{k}(\omega, F)$ to be the centre of the smallest ball circumscribing $F \in \mathcal{P}_{k}$. From (2.2) we have the following covariance property:

$$
\pi_{k}(\omega, F)=\pi_{k}(\omega-y, F-y)+y, \quad \omega \in N, F \in \mathcal{P}_{k} .
$$

As the factorial moment measures of a Poisson process are absolutely continuous with respect to Lebesgue measure, it can easily be shown that the points $\pi_{k}(N, F), F \in \delta_{k}(N)$, are almost surely mutually different. Moreover, for P-almost every $\omega$,

$$
\omega_{k}:=\sum_{F \in \delta_{k}(\omega)} \delta_{\pi_{k}(\omega, F)}
$$

is an element of $N^{\prime}$. For $\omega_{k} \in N^{\prime}$ and $x \in \omega_{k}$, there exists a unique $k$-face, $C_{k}(\omega, x) \in \wp_{k}(\omega)$, defined by the equivalence

$$
C_{k}(\omega, x)=F \quad \Longleftrightarrow \quad \pi_{k}(\omega, F)=x
$$


In all other cases, we define $C_{k}(\omega, x):=\{x\}$. For points $x \in \mathbb{R}^{d}$ in the relative interior of some $F \in \wp_{k}(\omega)$, we define $\pi_{k}(\omega, x):=\pi_{k}\left(\omega, F_{k}(\omega, x)\right)$. Otherwise, we let $\pi_{k}(\omega, x):=x$. From (2.4) we have

$$
\begin{array}{ll}
C_{k}(\omega, x)=C_{k}(\omega-x, 0)+x, & \omega \in N, x \in \mathbb{R}^{d}, \\
\pi_{k}(\omega, x)=\pi_{k}(\omega-x, 0)+x, & \omega \in N, x \in \mathbb{R}^{d} .
\end{array}
$$

We often write $F_{k}(x) \equiv F_{k}(N, x), C_{k}(x) \equiv C_{k}(N, x), \pi_{k}(x) \equiv \pi_{k}(N, x)$, and $\pi_{k}(F) \equiv$ $\pi_{k}(N, F)$.

We now define $N_{k}$, the point process of centres of $k$-faces, by $N_{k}(\omega):=\omega_{k}$ for $\omega_{k} \in N^{\prime}$ and $N_{k}(\omega):=0$ otherwise. By (2.7), $N_{k}$ is stationary. Note that $N_{d}=N$. The distribution of the cell $C_{k}(0)$ under the Palm probability measure $\mathrm{P}_{N_{k}}^{0}$ is that of the typical k-face of the Voronoi tessellation. Actually, we interpret $\mathrm{P}_{N_{k}}^{0}$ itself as the distribution of $N$ as seen from a typical $k$-face. As expected, the Palm measure of $N_{k}$ is closely related to the Palm measure of $M_{k}$.

Proposition 2.1. For all measurable functions $g: N \rightarrow[0, \infty)$, we have

$$
\begin{aligned}
\mathrm{E}_{M_{k}}\left[g\left(N-\pi_{k}(0)\right)\right] & =\mathrm{E}_{N_{k}}\left[\mathscr{H}^{k}\left(C_{k}(0)\right) g(N)\right], \\
\mathrm{E}_{N_{k}}[g(N)] & =\mathrm{E}_{M_{k}}\left[\mathscr{H}^{k}\left(F_{k}(0)\right)^{-1} g\left(N-\pi_{k}(0)\right)\right] .
\end{aligned}
$$

Proof. Both formulae are basically known. The first can be most easily proved with the help of the exchange formula of [9]. To prove the second we can apply the first with $g$ replaced with $g \mathscr{H}^{k}\left(C_{k}(0)\right)^{-1}$. Then (2.9) follows from

$$
C_{k}\left(N-\pi_{k}(0), 0\right)=F_{k}(0)-\pi_{k}(0) \quad \mathrm{P}_{M_{k}} \text {-almost surely. }
$$

By (2.8), the intensity, $\lambda_{k}$, of $N_{k}$ is related to the intensity, $\mu_{k}$, of $M_{k}$ by the intuitively obvious formula

$$
\mu_{k}=\lambda_{k} \mathrm{E}_{N_{k}}^{0}\left[\mathscr{H}^{k}\left(C_{k}(0)\right)\right] .
$$

Equation (2.9) tells us that the Palm measure $\mathrm{P}_{N_{k}}$ is an area-debiased version of the Palm measure $\mathrm{P}_{M_{k}}$.

The next proposition is basically a well-known fact about general stationary face-to-face tessellations. For more details, we refer the reader to Theorem 5.1 of [6], which treats the case of shift-invariant functions.

Proposition 2.2. For any measurable function $g: N \times N \rightarrow[0, \infty)$ and any $j, 0 \leq j<k \leq$ $d$, we have

$$
\mathrm{E}_{N_{j}}\left[\sum_{F \in \mathcal{S}_{k}(N), C_{j}(0) \subset F} g\left(N, N-\pi_{k}(F)\right)\right]=\mathrm{E}_{N_{k}}\left[\sum_{G \in \mathcal{S}_{j}(N), G \subset C_{k}(0)} g\left(N-\pi_{j}(G), N\right)\right] .
$$

As $M_{0}=N_{0}$ has a finite intensity, it follows from the version of this proposition with $j=0$ and $g:=1$ that the intensity of $N_{k}$ is finite for all $k \in\{0, \ldots, d\}$. The values of this intensity 
in the cases $d=2$ and $d=3$ can be found in, e.g. [10, p. 262]. The following special case will be important in Section 5.

Corollary 2.1. For any measurable function $f: N \rightarrow[0, \infty)$, we have

$$
\mathrm{E}_{N_{0}}\left[\sum_{F \in \oiint_{1}(N), 0 \in F} f\left(N-\pi_{1}(F)\right)\right]=2 \mathrm{E}_{N_{1}}[f(N)] .
$$

\section{Proof of Theorem 1.1}

We first need to introduce further notation. Let $k \in\{0, \ldots, d\}$, let $\omega \in N$, and let $x$ be a point in the relative interior of some $F \in \delta_{k}(\omega)$. For $k \leq d-1$, the number

$$
R_{k}^{\prime}(\omega, x):=\left|X_{k, 0}(\omega, x)-Z_{k}(\omega, x)\right|
$$

is positive, and we can thus define the unit vectors

$$
U_{k, i}(\omega, x):=\frac{X_{k, i}(\omega, x)-Z_{k}(\omega, x)}{R_{k}^{\prime}(\omega, x)}, \quad i=0, \ldots, d-k .
$$

For $k=d$, we define $R_{d}^{\prime}(\omega, x):=0$ and $U_{d, 0}(\omega, x):=0$. Furthermore, for $k \geq 1$ we define

$$
R_{k}^{\prime \prime}(\omega, x):=\left|x-Z_{k}(\omega, x)\right|
$$

and, given that $R_{k}^{\prime \prime}(\omega, x)>0$, the unit vector

$$
U_{k}(\omega, x):=\frac{Z_{k}(\omega, x)-x}{R_{k}^{\prime \prime}(\omega, x)} .
$$

In the exceptional case in which $R_{k}^{\prime \prime}(\omega, x)=0$, we choose $U_{k}(\omega, x)$ to equal some fixed unit vector. For $k=0$, we define $R_{0}^{\prime \prime}(\omega, x):=0$ and $U_{0}(\omega, x):=0$. For points $x \in \mathbb{R}^{d}$ that are not in the relative interior of some $k$-face, we let $R_{k}^{\prime}(\omega, x)=R_{k}^{\prime \prime}(\omega, x) \equiv 0$ and choose $U_{k, 0}(\omega, x), \ldots, U_{k, d-k}(\omega, x), U_{k}(\omega, x)$ to be fixed unit vectors. Exceptions are the cases in which $k=0$ and $k=d$, where we define $U_{0}(\omega, x):=0$ and $U_{d, 0}(\omega, x):=0$, respectively. The mappings $R_{k}^{\prime}, R_{k}^{\prime \prime}$, and $U_{k, i}$ have the same invariance property as $R_{k}$ in (2.3).

We can now rewrite the random variables occurring in Theorem 1.1 as follows: $R_{k} \equiv$ $R_{k}(N, 0), R_{k}^{\prime} \equiv R_{k}^{\prime}(N, 0), R_{k}^{\prime \prime} \equiv R_{k}^{\prime \prime}(N, 0), U_{k, i} \equiv U_{k, i}(N, 0)$ for $i=0, \ldots, d-k$, and $U_{k} \equiv U_{k}(N, 0)$. For ease of exposition of the proof of the theorem, we now assume that $1 \leq k \leq d-1$. The (well-known) case in which $k=d$ is easy to treat while the (somewhat easier) case in which $k=0$ can be proved similarly. Our proof is similar to those of Theorem 7.2 of [6] and Satz 6.2.4 of [10]. Let $B:=B(0,1)$. For all measurable functions $h: N \rightarrow[0, \infty)$, we have

$$
\begin{aligned}
& \kappa_{d} \mathrm{E}_{M_{k}}[h(N)]=\mathrm{E}\left[\int_{B} h(N-y) M_{k}(\mathrm{~d} y)\right] \\
&=\frac{1}{(d-k+1) !} \mathrm{E}\left[\sum_{x_{0}, \ldots, x_{d-k} \in N}^{*} \int h(N-y) 1_{\left\{y \in F\left(x_{0}, \ldots, x_{d-k}\right) \cap B\right\}}\right. \\
&\left.\times 1_{\left\{B^{0}\left(y,\left|y-x_{0}\right|\right) \cap N=\varnothing\right\}} \mathscr{H}^{k}(\mathrm{~d} y)\right],
\end{aligned}
$$


where ' $*$ ' indicates that the summation is over pairwise-different points of $N$. Using an iterated version of Mecke's fundamental formula for Poisson processes (see Satz 3.1 of [3]) and letting

$$
c:=\lambda^{d-k+1}\left(\kappa_{d}(d-k+1) !\right)^{-1},
$$

we obtain

$$
\begin{array}{r}
\mathrm{E}_{M_{k}}[h(N)]=c \mathrm{E}\left[\int \cdots \int h\left(\left(N \cup\left\{x_{0}, \ldots, x_{d-k}\right\}\right)-y\right) 1_{\left\{y \in F\left(x_{0}, \ldots, x_{d-k}\right) \cap B\right\}}\right. \\
\left.\times 1_{\left\{N \cap B^{0}\left(y,\left|y-x_{0}\right|\right)=\varnothing\right\}} \mathcal{H}^{k}(\mathrm{~d} y) \mathrm{d} x_{0} \cdots \mathrm{d} x_{d-k}\right] .
\end{array}
$$

For $\omega \in N, t \geq 0$, and $y \in \mathbb{R}^{d}$, we define

$$
\omega^{t}:=\omega \cap\left(\mathbb{R}^{d} \backslash B(0, t)\right) \text { and } \Psi(\omega, y):=\left\{U_{0}(\omega, y), \ldots, U_{d-k}(\omega, y)\right\},
$$

where on the right-hand side of the latter definition (and below) we omit the indices referring to the dimension of the faces. We apply the previous result to

$$
h(\omega):=g_{1}\left(\omega^{R(\omega, 0)}\right) g_{2}\left(R(\omega, 0), R^{\prime \prime}(\omega, 0)\right) w_{1}(\Psi(\omega, 0)) w_{2}(U(\omega, 0)),
$$

for suitable measurable, nonnegative functions $g_{1}, g_{2}, w_{1}$, and $w_{2}$. Using covariance and invariance properties as in (2.2) and (2.3), we obtain

$$
\begin{aligned}
\mathrm{E}_{M_{k}}[h(N)]=c \mathrm{E}\left[\int \cdots \int\right. & 1_{\left\{y \in F\left(x_{0}, \ldots, x_{d-k}\right) \cap B\right\}} 1_{\left\{N \cap B^{0}\left(y,\left|y-x_{0}\right|\right)=\varnothing\right\}} \\
& \times g_{1}\left((N-y) R\left(N \cup\left\{x_{0}, \ldots, x_{d-k}\right\}, y\right)\right) \\
& \times g_{2}\left(R\left(N \cup\left\{x_{0}, \ldots, x_{d-k}\right\}, y\right), R^{\prime \prime}\left(N \cup\left\{x_{0}, \ldots, x_{d-k}\right\}, y\right)\right) \\
& \times w_{1}\left(\Psi\left(N \cup\left\{x_{0}, \ldots, x_{d-k}\right\}, y\right)\right) \\
& \left.\times w_{2}\left(U\left(N \cup\left\{x_{0}, \ldots, x_{d-k}\right\}, y\right)\right) \mathscr{H}^{k}(\mathrm{~d} y) \mathrm{d} x_{0} \cdots \mathrm{d} x_{d-k}\right] .
\end{aligned}
$$

Assume that $x_{0}, \ldots, x_{d-k}$ are in general position. Taking $y \in F\left(x_{0}, \ldots, x_{d-k}\right)$ and assuming that

$$
N \cap B\left(y,\left|y-x_{0}\right|\right)=\varnothing,
$$

we have

$$
\begin{aligned}
R\left(N \cup\left\{x_{0}, \ldots, x_{d-k}\right\}, y\right) & =\left|x_{0}-y\right|, \\
R^{\prime \prime}\left(N \cup\left\{x_{0}, \ldots, x_{d-k}\right\}, y\right) & =\left|z\left(x_{0}, \ldots, x_{d-k}\right)-y\right|, \\
\Psi\left(N \cup\left\{x_{0}, \ldots, x_{d-k}\right\}, y\right) & =\tilde{\Psi}\left(x_{0}, \ldots, x_{d-k}\right), \\
U\left(N \cup\left\{x_{0}, \ldots, x_{d-k}\right\}, y\right) & =\tilde{U}\left(x_{0}, \ldots, x_{d-k}, y\right),
\end{aligned}
$$

where

$$
\begin{gathered}
\tilde{\Psi}\left(x_{0}, \ldots, x_{d-k}\right):=\left\{\frac{x_{0}-z\left(x_{0}, \ldots, x_{d-k}\right)}{\left|x_{0}-z\left(x_{0}, \ldots, x_{d-k}\right)\right|}, \ldots, \frac{x_{d-k}-z\left(x_{0}, \ldots, x_{d-k}\right)}{\left|x_{d-k}-z\left(x_{0}, \ldots, x_{d-k}\right)\right|}\right\}, \\
\tilde{U}\left(x_{0}, \ldots, x_{d-k}, y\right):=\frac{z\left(x_{0}, \ldots, x_{d-k}\right)-y}{\left|z\left(x_{0}, \ldots, x_{d-k}\right)-y\right|} .
\end{gathered}
$$


(Here we take $\tilde{U}\left(x_{0}, \ldots, x_{d-k}, y\right)$ to equal some fixed unit vector if $z\left(x_{0}, \ldots, x_{d-k}\right)=y$. If $x_{0}, \ldots, x_{d-k}$ are not in general position, then all of these functions can be defined arbitrarily.) It follows that

$$
\begin{aligned}
\mathrm{E}_{M_{k}}[h(N)]=c \mathrm{E}\left[\int \cdots \int\right. & 1_{\left\{y \in F\left(x_{0}, \ldots, x_{d-k}\right) \cap B\right\}} 1_{\left\{N \cap B^{0}\left(y,\left|y-x_{0}\right|\right)=\varnothing\right\}} \\
& \times g_{1}\left((N-y)^{\left|x_{0}-y\right|}\right) g_{2}\left(\left|x_{0}-y\right|,\left|z\left(x_{0}, \ldots, x_{d-k}\right)-y\right|\right) \\
& \times w_{1}\left(\tilde{\Psi}\left(x_{0}, \ldots, x_{d-k}\right)\right) \\
& \left.\times w_{2}\left(\tilde{U}\left(x_{0}, \ldots, x_{d-k}, y\right)\right) \mathscr{H}^{k}(\mathrm{~d} y) \mathrm{d} x_{0} \cdots \mathrm{d} x_{d-k}\right] .
\end{aligned}
$$

Since $N$ is stationary and has independent increments, we obtain

$$
\begin{aligned}
\mathrm{E}_{M_{k}}[h(N)]=c \int \cdots \int & 1_{\left\{y \in F\left(x_{0}, \ldots, x_{d-k}\right) \cap B\right\}} \exp \left[-\lambda \kappa_{d}\left|y-x_{0}\right|^{d}\right] \\
& \times \mathrm{E}\left[g_{1}\left(N^{\left|x_{0}-y\right|}\right)\right] g_{2}\left(\left|x_{0}-y\right|,\left|z\left(x_{0}, \ldots, x_{d-k}\right)-y\right|\right) \\
& \times w_{1}\left(\tilde{\Psi}\left(x_{0}, \ldots, x_{d-k}\right)\right) \\
& \times w_{2}\left(\tilde{U}\left(x_{0}, \ldots, x_{d-k}, y\right)\right) \mathcal{H}^{k}(\mathrm{~d} y) \mathrm{d} x_{0} \cdots \mathrm{d} x_{d-k} .
\end{aligned}
$$

Following [10, pp. 260-261], we next apply the Blaschke-Petkantschin formula (see, e.g. Satz 7.2.1 of [10]) and Satz 7.2.2 of [10]. Recalling the definitions of the functions $\tilde{\Psi}$ and $\tilde{U}$, for a suitable constant $c^{\prime}>0$ we obtain

$$
\begin{aligned}
\mathrm{E}_{M_{k}}[h(N)]=c^{\prime} \int \cdots \int & 1_{\left\{y \in\left(z+\vartheta L^{\perp}\right) \cap B, z \in \vartheta\left(y_{0}+L\right), r \geq 0, y_{0} \in L^{\perp}\right\}} \\
& \times \mathrm{E}\left[g_{1}\left(N^{\sqrt{|y-z|^{2}+r^{2}}}\right)\right] \\
& \times g_{2}\left(\sqrt{|y-z|^{2}+r^{2}},|z-y|\right) w_{1}\left(\left\{u_{0}, \ldots, u_{d-k}\right\}\right) \\
& \times w_{2}\left(\frac{z-y}{|z-y|}\right) \exp \left[-\lambda \kappa_{d}\left(\sqrt{|y-z|^{2}+r^{2}}\right)^{d}\right] r^{d(d-k)-1} \\
& \times \Delta_{d-k}\left(u_{0}, \ldots, u_{d-k}\right)^{k+1} \\
& \times \mathscr{H}^{k}(\mathrm{~d} y) \mathbb{S}_{\vartheta L}\left(\mathrm{~d} u_{0}\right) \cdots \mathbb{S}_{\vartheta L}\left(\mathrm{~d} u_{d-k}\right) \mathrm{d} r \mathscr{H}^{d-k}(\mathrm{~d} z) \mathscr{H}^{k}\left(\mathrm{~d} y_{0}\right) v(\mathrm{~d} \vartheta)
\end{aligned}
$$

where $L \subset \mathbb{R}^{d}$ is some fixed $(d-k)$-dimensional linear subspace and $\mathbb{S}_{\vartheta L}$ is the uniform distribution on the unit sphere in the linear subspace $\vartheta L$. We take

$$
g_{2}(a, b):=g_{3}(a) g_{4}\left(\left(a^{2}-b^{2}\right) / a^{2}\right), \quad 0 \leq b<a,
$$


for measurable functions $g_{3}, g_{4}:[0, \infty) \rightarrow[0, \infty)$. Noting that $\Delta_{d-k}(\cdot),|\cdot|$, and $\mathscr{H}^{i}(\cdot)$ are invariant under rotations, and replacing $\left(u_{0}, \ldots, u_{d-k}, y, z\right)$ with $\left(\vartheta u_{0}, \ldots, \vartheta u_{d-k}, \vartheta y, \vartheta z\right)$, we arrive at

$$
\begin{aligned}
\mathrm{E}_{M_{k}}[h(N)]=c^{\prime} \int \ldots \int & 1_{\left\{y \in\left(z+L^{\perp}\right) \cap B, z \in y_{0}+L, r \geq 0, y_{0} \in L^{\perp}\right\}} \\
& \times \mathrm{E}\left[g_{1}\left(N^{\sqrt{|y-z|^{2}+r^{2}}}\right)\right] g_{3}\left(\sqrt{|y-z|^{2}+r^{2}}\right) \\
& \times g_{4}\left(\frac{r^{2}}{|y-z|^{2}+r^{2}}\right) w_{1}\left(\left\{\vartheta u_{0}, \ldots, \vartheta u_{d-k}\right\}\right) \\
& \times w_{2}\left(\frac{\vartheta(z-y)}{|z-y|}\right) \exp \left[-\lambda \kappa_{d}\left(\sqrt{|y-z|^{2}+r^{2}}\right)^{d}\right] r^{d(d-k)-1} \\
& \times \Delta_{d-k}\left(u_{0}, \ldots, u_{d-k}\right)^{k+1} \\
& \times \mathscr{H}^{k}(\mathrm{~d} y) \mathbb{S}_{L}\left(\mathrm{~d} u_{0}\right) \cdots \mathbb{S}_{L}\left(\mathrm{~d} u_{d-k}\right) \mathrm{d} r \mathscr{H}^{d-k}(\mathrm{~d} z) \mathscr{H}^{k}\left(\mathrm{~d} y_{0}\right) v(\mathrm{~d} \vartheta) \\
=c^{\prime} \int & 1_{\left\{x \in L^{\perp}, z-x \in B, z \in y_{0}+L, r \geq 0, y_{0} \in L^{\perp}\right\}} \mathrm{E}\left[g_{1}\left(N^{\sqrt{|x|^{2}+r^{2}}}\right)\right] \\
& \times g_{3}\left(\sqrt{|x|^{2}+r^{2}}\right) g_{4}\left(\frac{r^{2}}{|x|^{2}+r^{2}}\right) w_{1}\left(\left\{\vartheta u_{0}, \ldots, \vartheta u_{d-k}\right\}\right) \\
& \times w_{2}\left(\frac{\vartheta x}{|x|}\right) \exp \left[-\lambda \kappa_{d}\left(\sqrt{|x|^{2}+r^{2}}\right)^{d}\right] r^{d(d-k)-1} \\
& \times \Delta_{d-k}\left(u_{0}, \ldots, u_{d-k}\right)^{k+1} \mathscr{H}^{k}(\mathrm{~d} x) \mathbb{S}_{L}\left(\mathrm{~d} u_{0}\right) \ldots \mathbb{S}_{L}\left(\mathrm{~d} u_{d-k}\right) \\
& \times \mathrm{d} r \mathscr{H}^{d-k}(\mathrm{~d} z) \mathscr{H}^{k}\left(\mathrm{~d} y_{0}\right) v(\mathrm{~d} \vartheta), \\
\ldots &
\end{aligned}
$$

where we have used the change of variable $y=z-x$ to obtain the second equality. For any fixed $x \in \mathbb{R}^{d}$, it can easily be shown that

$$
\iint 1_{\left\{z-x \in B, z \in y_{0}+L, y_{0} \in L^{\perp}\right\}} \mathscr{H}^{d-k}(\mathrm{~d} z) \mathscr{H}^{k}\left(\mathrm{~d} y_{0}\right)=\kappa_{d} .
$$

Hence,

$$
\begin{aligned}
\mathrm{E}_{M_{k}}[h(N)]=\kappa_{d} c^{\prime} \int \ldots \int & 1_{\left\{x \in L^{\perp}, r \geq 0\right\}} \mathrm{E}\left[g_{1}\left(N^{\sqrt{|x|^{2}+r^{2}}}\right)\right] g_{3}\left(\sqrt{|x|^{2}+r^{2}}\right) \\
& \times g_{4}\left(\frac{r^{2}}{|x|^{2}+r^{2}}\right) w_{1}\left(\left\{\vartheta u_{0}, \ldots, \vartheta u_{d-k}\right\}\right) \\
& \times w_{2}\left(\frac{\vartheta x}{|x|}\right) \exp \left[-\lambda \kappa_{d}\left(\sqrt{|x|^{2}+r^{2}}\right)^{d}\right] \\
& \times r^{d(d-k)-1} \Delta_{d-k}\left(u_{0}, \ldots, u_{d-k}\right)^{k+1} \\
& \times \mathscr{H}^{k}(\mathrm{~d} x) \mathbb{S}_{L}\left(\mathrm{~d} u_{0}\right) \cdots \mathbb{S}_{L}\left(\mathrm{~d} u_{d-k}\right) \mathrm{d} r v(\mathrm{~d} \vartheta) .
\end{aligned}
$$


Using polar coordinates in $L^{\perp}$ gives

$$
\begin{aligned}
\mathrm{E}_{M_{k}}[h(N)]= & c^{\prime \prime} \int_{0}^{\infty} \int_{0}^{\infty} \mathrm{E}\left[g_{1}\left(N^{\sqrt{s^{2}+r^{2}}}\right)\right] g_{3}\left(\sqrt{s^{2}+r^{2}}\right) g_{4}\left(\frac{r^{2}}{r^{2}+s^{2}}\right) \\
& \times \exp \left[-\lambda \kappa_{d}\left(\sqrt{s^{2}+r^{2}}\right)^{d}\right] r^{d(d-k)-1} s^{k-1} \mathrm{~d} r \mathrm{~d} s \\
\times \int \cdots \int & w_{1}\left(\left\{\vartheta u_{0}, \ldots, \vartheta u_{d-k}\right\}\right) w_{2}(\vartheta u) \\
& \times \Delta_{d-k}\left(u_{0}, \ldots, u_{d-k}\right)^{k+1} \mathbb{S}_{L}\left(\mathrm{~d} u_{0}\right) \cdots \mathbb{S}_{L}\left(\mathrm{~d} u_{d-k}\right) \mathbb{S}_{L^{\perp}}(\mathrm{d} u) v(\mathrm{~d} \vartheta),
\end{aligned}
$$

for a suitable constant $c^{\prime \prime}$. It remains to treat the first double integral. Here, using the substitution $(s, r)=(v \sqrt{1-t}, v \sqrt{t}), t \in[0,1], v \in[0, \infty)$, with Jacobian $v / 2 \sqrt{t(1-t)}$, we find that the integral is proportional to

$$
\int_{0}^{\infty} \mathrm{E}\left[g_{1}\left(N^{v}\right)\right] g_{3}(v) v^{d(d-k)+k-1} \mathrm{e}^{-\lambda \kappa_{d} v^{d}} \mathrm{~d} v \int_{0}^{1} t^{d(d-k) / 2-1}(1-t)^{k / 2-1} g_{4}(t) \mathrm{d} t .
$$

The substitution $z=v^{d}$ completes the proof of the theorem.

\section{Typical faces of a Poisson-Voronoi tessellation}

In this section we deal with the distribution of the area of the typical $k$-face and its neighbours. Our basic general tool is Proposition 2.1. We first need to establish some results that hold under $\mathrm{P}_{k}^{0}$.

Proposition 4.1. Let $k \in\{1, \ldots, d\}$. Then $\left(\mathscr{H}^{k}\left(F_{k}(0)\right), R_{k}^{\prime}, R_{k}^{\prime \prime}\right)$ and $\Psi_{k}$ are independent under the Palm probability measure $\mathrm{P}_{k}^{0}$.

Proof. For $\omega \in N, r, r^{\prime \prime} \geq 0$, and $u, v \in S^{d-1}$, we define

$$
\rho\left(\omega, r, r^{\prime \prime}, u, v\right):=\sup \left\{t \geq 0: B^{0}\left(t v, \sqrt{r^{2}+t^{2}-2 r^{\prime \prime} t\langle u, v\rangle}\right) \cap \omega^{r}=\varnothing\right\} .
$$

Recall that $\omega^{r}:=\omega \cap\left(\mathbb{R}^{d} \backslash B(0, r)\right)$. An interpretation of this function will be given below.

In the following we will always assume that 0 is in the relative interior of some $k$-face. By definition, the linear hull of the $k$-face $F_{k}(0)$ is then given by

$$
G_{k}:=\operatorname{Span}\left(U_{k, 0}, \ldots, U_{k, d-k}\right)^{\perp} .
$$

Take $v \in G_{k} \cap S^{d-1}$ and recall that $X_{k, 0}$ is one of the neighbours of $F_{k}(0)$. Then

$$
\begin{aligned}
\left|X_{k, 0}-t v\right|^{2} & =\left|R_{k}^{\prime} U_{k, 0}+R_{k}^{\prime \prime} U_{k}-t v\right|^{2} \\
& =R_{k}^{\prime 2}+\left|R_{k}^{\prime \prime} U_{k}-t v\right|^{2} \\
& =R_{k}^{\prime 2}+R_{k}^{\prime \prime 2}+t^{2}-2 R_{k}^{\prime \prime} t\left\langle U_{k}, v\right\rangle \\
& =R_{k}^{2}+t^{2}-2 R_{k}^{\prime \prime} t\left\langle U_{k}, v\right\rangle .
\end{aligned}
$$

Hence, we have the equivalence

$$
t v \in F_{k}(0) \Longleftrightarrow \rho\left(N, R_{k}, R_{k}^{\prime \prime}, U_{k}, v\right) \geq t,
$$


according to which $\rho\left(N, R_{k}, R_{k}^{\prime \prime}, U_{k}, \cdot\right)$ can be interpreted as the radial function of $F_{k}(0)$. It follows that

$$
\mathscr{H}^{k}\left(F_{k}(0)\right)=A_{G_{k}}\left(N, R_{k}, R_{k}^{\prime \prime}, U_{k}\right),
$$

where, for a $k$-dimensional linear subspace $S$,

$$
A_{S}\left(\omega, r, r^{\prime \prime}, u\right):=\frac{1}{k} \int_{S^{d-1} \cap S} \rho\left(\omega, r, r^{\prime \prime}, u, v\right)^{k} \mathscr{H}^{k-1}(\mathrm{~d} v) .
$$

We now apply Theorem 1.1, to obtain, for measurable, nonnegative functions $f_{1}, f_{2}$, and $f_{3}$ with suitable domains,

$$
\begin{aligned}
\mathrm{E}_{M_{k}}^{0}\left[f_{1}\left(\mathscr{H}^{k}\left(F_{k}(0)\right)\right)\right. & \left.f_{2}\left(R_{k}, R_{k}^{\prime \prime}\right) f_{3}\left(\Psi_{k}\right)\right] \\
=c_{k}^{-1} \int \cdots \int & f_{1}\left(A_{\vartheta L^{\perp}}\left(\omega, r, r^{\prime \prime}, \vartheta u\right)\right) f_{2}\left(r, r^{\prime \prime}\right) f_{3}\left(\left\{\vartheta u_{0}, \ldots, \vartheta u_{d-k}\right\}\right) \\
& \times \Delta_{d-k}\left(u_{0}, \ldots, u_{d-k}\right)^{k+1} \\
& \times \mathbb{S}_{L}\left(\mathrm{~d} u_{0}\right) \cdots \mathbb{S}_{L}\left(\mathrm{~d} u_{d-k}\right) \mathbb{S}_{L^{\perp}}(\mathrm{d} u) v(\mathrm{~d} \vartheta) \Pi^{r}(\mathrm{~d} \omega) \mathrm{P}_{k}^{0}\left(\left(R, R^{\prime \prime}\right) \in \mathrm{d}\left(r, r^{\prime \prime}\right)\right),
\end{aligned}
$$

where $\Pi^{r}$ is the distribution of $N^{r}$ under P. For any fixed $r, r^{\prime \prime} \geq 0$, we have the invariance property $\rho\left(\vartheta \omega, r, r^{\prime \prime}, \vartheta u, \vartheta v\right)=\rho\left(\omega, r, r^{\prime \prime}, u, v\right)$ for $\omega \in N, u, v \in S^{d-1}$, and $\vartheta \in S O_{d}$. As $\Pi^{r}$ is invariant under rotations, it follows that

$$
\begin{aligned}
\iint f_{1}\left(A_{\vartheta L^{\perp}}\left(\omega, r, r^{\prime \prime}, \vartheta u\right)\right) \mathbb{S}_{L^{\perp}}(\mathrm{d} u) \Pi^{r}(\mathrm{~d} \omega) \\
=\iint f_{1}\left(A_{\vartheta L^{\perp}}\left(\vartheta \omega, r, r^{\prime \prime}, \vartheta u\right)\right) \mathbb{S}_{L^{\perp}}(\mathrm{d} u) \Pi^{r}(\mathrm{~d} \omega) \\
=\iint f_{1}\left(A_{L^{\perp}}\left(\omega, r, r^{\prime \prime}, u\right)\right) \mathbb{S}_{L^{\perp}}(\mathrm{d} u) \Pi^{r}(\mathrm{~d} \omega) \\
=: f_{1}^{*}\left(r, r^{\prime \prime}\right) .
\end{aligned}
$$

Inserting this result into (4.1), we obtain

$$
\begin{aligned}
\mathrm{E}_{M_{k}}^{0}[ & \left.f_{1}\left(\mathscr{H}^{k}\left(F_{k}(0)\right)\right) f_{2}\left(R_{k}, R_{k}^{\prime \prime}\right) f_{3}\left(\Psi_{k}\right)\right] \\
= & c_{k}^{-1} \int \cdots \int f_{1}^{*}\left(r, r^{\prime \prime}\right) f_{2}\left(r, r^{\prime \prime}\right) f_{3}\left(\left\{\vartheta u_{0}, \ldots, \vartheta u_{d-k}\right\}\right) \Delta_{d-k}\left(u_{0}, \ldots, u_{d-k}\right)^{k+1} \\
& \quad \times \mathbb{S}_{L}\left(\mathrm{~d} u_{0}\right) \cdots \mathbb{S}_{L}\left(\mathrm{~d} u_{d-k}\right) v(\mathrm{~d} \vartheta) \mathrm{P}_{k}^{0}\left(\left(R, R^{\prime \prime}\right) \in \mathrm{d}\left(r, r^{\prime \prime}\right)\right) \\
& =\mathrm{E}_{M_{k}}^{0}\left[f_{1}^{*}\left(R_{k}, R_{k}^{\prime \prime}\right) f_{2}\left(R_{k}, R_{k}^{\prime \prime}\right) f_{3}\left(\Psi_{k}\right)\right] \\
= & \mathrm{E}_{M_{k}}^{0}\left[f_{1}^{*}\left(R_{k}, R_{k}^{\prime \prime}\right) f_{2}\left(R_{k}, R_{k}^{\prime \prime}\right)\right] \mathrm{E}_{M_{k}}^{0}\left[f_{3}\left(\Psi_{k}\right)\right],
\end{aligned}
$$

where we have again used Theorem 1.1. Using this and specializing to the case in which $f_{3}:=1$ yields the assertion.

The preceding proof yields more detailed, though not very explicit, information on the distribution of $\left(\mathscr{H}^{k}\left(F_{k}(0)\right), R_{k}, R_{k}^{\prime \prime}\right)$ under $\mathrm{P}_{k}^{0}$. 
Corollary 4.1. For all $k \in\{1, \ldots, d\}$, we $\mathrm{P}_{k}^{0}$-almost surely have

$$
\mathrm{P}_{k}^{0}\left(\mathcal{H}^{k}\left(F_{k}(0)\right) \in \cdot \mid R_{k}, R_{k}^{\prime \prime}\right)=\iint 1_{\left\{A_{L^{\perp}}\left(\omega, R_{k}, R_{k}^{\prime \prime}, u\right) \in \cdot\right\}^{\perp}} \mathbb{S}_{L^{\perp}}(\mathrm{d} u) \Pi^{R_{k}}(\mathrm{~d} \omega) .
$$

For $x \in N_{k}$, we let $\rho_{k}(x) \equiv p_{k}(N, x)$ denote the distance of $x$ from $N$ and $V_{k, 0}(x), \ldots$, $V_{k, d-k}(x)$ the unit vectors such that $\rho_{k}(x) V_{k, 0}(x), \ldots, \rho_{k}(x) V_{k, d-k}(x)$ are the neighbours of the $k$-face $C_{k}(x)$ (see (2.5)). We let $\Phi_{k}(x) \equiv \Phi_{k}(N, x):=\left\{V_{k, 0}(x), \ldots, V_{k, d-k}(x)\right\}$. For $x \notin N_{k}$, we give $\rho_{k}(x)$ and $\Phi_{k}(x)$ some fixed values.

Theorem 4.1. For any $k \in\{1, \ldots, d\}$, the following assertions hold under $\mathrm{P}_{N_{k}}^{0}$.

(i) The random variables $\left(\mathscr{H}^{k}\left(C_{k}(0)\right), \rho_{k}(0)\right)$ and $\Phi_{k}(0)$ are independent.

(ii) The distribution of $\Phi_{k}(0)$ is $\mathbb{Q}_{k}\left(\cdot \times S^{d-1}\right)$, where $\mathbb{Q}_{k}$ is as given in (1.3).

Proof. We will use Proposition 4.1 together with (2.9). Assume that 0 is in the relative interior of a $k$-face. Then $\Phi_{k}\left(N-\pi_{k}(0), 0\right)=\Psi_{k}, \rho_{k}\left(N-\pi_{k}(0), 0\right)=R_{k}^{\prime}$, and $C_{k}\left(N-\pi_{k}(0), 0\right)=$ $F_{k}(0)-\pi_{k}(0)$. Applying (2.9) to measurable functions $h_{1}$ and $h_{2}$ (with suitable domains), we obtain

$$
\begin{aligned}
\lambda_{k} \mathrm{E}_{N_{k}}^{0} & {\left[h_{1}\left(\mathscr{H}^{k}\left(C_{k}(0)\right), \rho_{k}(0)\right) h_{2}\left(\Phi_{k}(0)\right)\right] } \\
& =\mu_{k} \mathrm{E}_{M_{k}}^{0}\left[\mathscr{H}^{k}\left(F_{k}(N, 0)\right)^{-1} h_{1}\left(\mathscr{H}^{k}\left(F_{k}(0)\right), R_{k}^{\prime}\right) h_{2}\left(\Psi_{k}\right)\right] \\
& =\mu_{k} \mathrm{E}_{M_{k}}^{0}\left[\mathscr{H}^{k}\left(F_{k}(N, 0)\right)^{-1} h_{1}\left(\mathscr{H}^{k}\left(F_{k}(0)\right), R_{k}^{\prime}\right)\right] \mathrm{E}_{M_{k}}^{0}\left[h_{2}\left(\Psi_{k}\right)\right] \\
& =\lambda_{k} \mathrm{E}_{N_{k}}^{0}\left[h_{1}\left(\mathscr{H}^{k}\left(C_{k}(0)\right), \rho_{k}(0)\right)\right] \mathrm{E}_{M_{k}}^{0}\left[h_{2}\left(\Psi_{k}\right)\right],
\end{aligned}
$$

where we have used Proposition 4.1 to obtain the second equality. Specializing to the case in which $h_{1}:=1$ yields (i) and, in view of Theorem 1.1(v), also (ii).

The above proof and Corollary 4.1 yield the following result.

Corollary 4.2. For all $k \in\{1, \ldots, d\}$, we have

$$
\begin{aligned}
\mathrm{P}_{N_{k}}^{0}\left(\left(\mathcal{H}^{k}\left(C_{k}(0)\right), \rho_{k}(0)\right) \in \cdot\right)=\frac{\mu_{k}}{\lambda_{k}} \iiint & A_{L^{\perp}}\left(\omega, r, r^{\prime \prime}, u\right)^{-1} 1_{\left\{\left(A_{L^{\perp}}\left(\omega, r, r^{\prime \prime}, u\right), \sqrt{r^{2}-r^{\prime \prime 2}}\right) \in \cdot\right\}} \\
& \times \mathbb{S}_{L^{\perp}}(\mathrm{d} u) \Pi^{r}(\mathrm{~d} \omega) \mathrm{P}_{k}^{0}\left(\left(R_{k}, R_{k}^{\prime \prime}\right) \in \mathrm{d}\left(r, r^{\prime \prime}\right)\right),
\end{aligned}
$$

where the distribution $\mathrm{P}_{k}^{0}\left(\left(R_{k}, R_{k}^{\prime \prime}\right) \in \cdot\right)$ is as given in Theorem 1.1.

Finally in this section, we establish a more detailed version of Proposition 4.1 that holds for $k=1$. For a point $x$ in the relative interior of some edge $F \in \varsigma_{1}(N)$, we define $U_{1}^{*}(x)$ as the unique vector in $\left\{-U_{1}(N, x), U_{1}(N, x)\right\}$ such that $-U_{1}^{*}(x)$ is lexicographically smaller than $U_{1}^{*}(x)$. Furthermore, we let $I_{1}(x)$ denote the $\{-1,1\}$-valued random variable satisfying

$$
U_{1}(N, x)=I_{1}(x) U_{1}^{*}(x)
$$

and $T^{\prime}(x)$ and $T^{\prime \prime}(x)$ the nonnegative random variables satisfying

$$
F_{1}(x)=\left[x-T^{\prime}(x) U_{1}^{*}(x), x+T^{\prime \prime}(x) U_{1}^{*}(x)\right] .
$$

Note that $T^{\prime}(x)+T^{\prime \prime}(x)=\mathscr{H}^{1}\left(F_{1}(x)\right)$ is the length of the edge $F_{1}(x)$. For all points $x$ that are not in the relative interior of some edge, we give $U_{1}^{*}(x), I_{1}(x), T^{\prime}(x)$, and $T^{\prime \prime}(x)$ some fixed 
values. Similarly to above, we use the abbreviations $T^{\prime} \equiv T^{\prime}(0), T^{\prime \prime} \equiv T^{\prime \prime}(0), I_{1} \equiv I_{1}(0)$, and $U_{1}^{*} \equiv U_{1}^{*}(0)$.

Together with Theorem 1.1, the following proposition provides a complete description of the joint distribution of the edge $\left[-T^{\prime} U_{1}^{*}, T^{\prime \prime} U_{1}^{*}\right]$ and its neighbours under $\mathrm{P}_{1}^{0}$. We denote by $V(a, s, t)$ the $d$-dimensional volume of the union of two balls with radii $s$ and $t$ whose centres are separated by a distance $a$.

Proposition 4.2. Under the Palm probability measure $\mathrm{P}_{1}^{0}$, the random variables $\Psi_{1}$ and $\left(T^{\prime}, T^{\prime \prime}, I_{1}, R_{1}, R_{1}^{\prime}\right)$ are independent and the random variables $T^{\prime}$ and $T^{\prime \prime}$ are conditionally independent given $\left(I_{1}, R_{1}, R_{1}^{\prime}\right)$. Furthermore, for all $t_{1}, t_{2}>0$, we almost surely have

$$
\begin{gathered}
\mathrm{P}_{1}^{0}\left(T^{\prime}>t_{1} \mid I_{1}, R_{1}, R_{1}^{\prime}\right)=\mathrm{e}^{\lambda \kappa_{d} R_{1}^{d}} \exp \left[-\lambda V\left(t_{1}, R_{1}, \sqrt{R_{1}^{2}+2 I_{1} R_{1}^{\prime \prime} t_{1}+t_{1}^{2}}\right)\right], \\
\mathrm{P}_{1}^{0}\left(T^{\prime \prime}>t_{2} \mid I_{1}, R_{1}, R_{1}^{\prime}\right)=\mathrm{e}^{\lambda \kappa_{d} R_{1}^{d}} \exp \left[-\lambda V\left(t_{2}, R_{1}, \sqrt{R_{1}^{2}-2 I_{1} R_{1}^{\prime \prime} t_{2}+t_{2}^{2}}\right)\right] .
\end{gathered}
$$

Proof. $\mathrm{P}_{1}^{0}$-almost surely, the point 0 is in the relative interior of some $F \in \varsigma_{1}(N)$ and

$$
\begin{aligned}
& \left\{T^{\prime}>t_{1}\right\}=\left\{N \cap B\left(-t_{1} U_{1}^{*}, \sqrt{R_{1}^{\prime 2}+\left(t_{1}+I_{1} R_{1}^{\prime \prime}\right)^{2}}\right) \cap\left(\mathbb{R}^{d} \backslash B\left(0, R_{1}\right)\right)=\varnothing\right\}, \\
& \left\{T^{\prime \prime}>t_{2}\right\}=\left\{N \cap B\left(t_{2} U_{1}^{*}, \sqrt{R_{1}^{\prime 2}+\left(t_{2}-I_{1} R_{1}^{\prime \prime}\right)^{2}}\right) \cap\left(\mathbb{R}^{d} \backslash B\left(0, R_{1}\right)\right)=\varnothing\right\} .
\end{aligned}
$$

It is easy to check that

$$
B\left(-t_{1} U_{1}^{*}, \sqrt{R_{1}^{\prime 2}+\left(t_{1}+I_{1} R_{1}^{\prime \prime}\right)^{2}}\right) \cap B\left(t_{2} U_{1}^{*}, \sqrt{R_{1}^{\prime 2}+\left(t_{2}-I_{1} R_{1}^{\prime \prime}\right)^{2}}\right) \cap\left(\mathbb{R}^{d} \backslash B\left(0, R_{1}\right)\right)=\varnothing .
$$

Assertions (i) and (iii) of Theorem 1.1 imply that

$$
\mathrm{P}\left(N \backslash B\left(0, R_{1}\right) \in \cdot \mid R_{1}, R_{1}^{\prime}, \Psi_{1}, I_{1}\right)=\mathrm{P}\left(N \backslash B\left(0, R_{1}\right) \in \cdot \mid R_{1}\right) \quad \mathrm{P}_{1}^{0} \text {-almost surely. }
$$

Hence, $T^{\prime}$ and $T^{\prime \prime}$ are conditionally independent given $\left(\Psi_{1}, I_{1}, R_{1}, R_{1}^{\prime}\right)$. The theorem also implies that

$$
\begin{aligned}
\mathrm{P}_{1}^{0}\left(T^{\prime}\right. & \left.>t_{1} \mid R_{1}, R_{1}^{\prime}, \Psi_{1}, I_{1}\right) \\
& =\exp \left[-\lambda \mathscr{H}^{d}\left(B\left(-t_{1} U_{1}^{*}, \sqrt{R_{1}^{2}+2 I_{1} R_{1}^{\prime \prime} t_{1}+t_{1}^{2}}\right) \backslash B\left(0, R_{1}\right)\right)\right] \\
& =\mathrm{e}^{\lambda \kappa_{d} R_{1}^{d}} \exp \left[-\lambda V\left(t_{1}, R_{1}, \sqrt{R_{1}^{2}+2 I_{1} R_{1}^{\prime \prime} t_{1}+t_{1}^{2}}\right)\right],
\end{aligned}
$$

where we have used the fact that $R_{1}^{\prime 2}+R_{1}^{\prime \prime 2}=R_{1}^{2}$. A similar formula holds for the conditional distribution of $T^{\prime \prime}$. In particular, we obtain the asserted formulae. Given $\Psi_{1}$, there are almost surely only two possible values for $U_{1}$. Hence, it follows easily from Theorem $1.1(\mathrm{v})$ that $\Psi_{1}$ and $I_{1}$ are independent. Using the fact that the above conditional probabilities depend only on $\left(I_{1}, R_{1}, R_{1}^{\prime \prime}\right)$, we obtain the remaining assertions using Theorem 1.1(i).

\section{The typical edge and its neighbours}

The topic of this, final, section is the Palm probability measure $\mathrm{P}_{N_{1}}^{0}$. It turns out that under this measure Theorem 4.1 (and Corollary 4.2) can be considerably improved. 


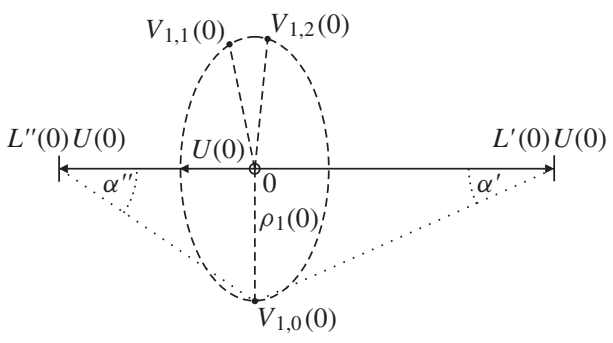

Figure 2: The situation under $\mathrm{P}_{N_{1}}^{0}$, for $d=3$.

First we introduce a convenient description of the typical edge $C_{1}(0)$ (see (2.5) for notation). For $x \in N_{1}$, we let $U(x)$ denote the unit vector orthogonal to the affine hull of $\Phi_{1}(x)$ such that $-U(x)$ is lexicographically smaller than $U(x)$. Let $L^{\prime}(x)$ and $L^{\prime \prime}(x), L^{\prime}(x) \leq L^{\prime \prime}(x)$, denote random variables such that

$$
C_{1}(x)=\left[L^{\prime}(x) U(x), L^{\prime \prime}(x) U(x)\right] .
$$

For $x \notin N_{1}$, we give $U(x)$ some fixed value and define $L^{\prime}(x)=L^{\prime \prime}(x):=0$. The case in which $d=3$ and $k=1$ is illustrated in Figure 2 (using the notation introduced just before Theorem 4.1).

By (2.10), we have $\mu_{1}=\lambda_{1} \mathrm{E}_{N_{1}}^{0}\left[L^{\prime \prime}(0)-L^{\prime}(0)\right]$. Since $2 \lambda_{1}=(d+1) \lambda_{0}$ (to see this, set $f:=1$ in Corollary 2.1) and $\lambda_{0}=\mu_{0}$, we obtain

$$
\mathrm{E}_{N_{1}}^{0}\left[L^{\prime \prime}(0)-L^{\prime}(0)\right]=\frac{\mu_{1}}{\lambda_{1}}=\frac{2 \mu_{1}}{(d+1) \lambda_{0}}=\frac{2 \mu_{1}}{(d+1) \mu_{0}} .
$$

Hence, (1.2) yields a formula for the mean length, $\mathrm{E}_{N_{1}}^{0}\left[L^{\prime \prime}(0)-L^{\prime}(0)\right]$, of the typical edge; see also [8, p. 285].

We now introduce the notation

$$
V^{*}(t, r, w):=\frac{\mathrm{d}}{\mathrm{d} t} V\left(t, r, \sqrt{t^{2}+r^{2}-2 \operatorname{tr} w}\right), \quad t, r \geq 0,|w| \leq 1 .
$$

More details on this function can be found in [8] and below. We define

$$
J\left(u_{0}, u_{1}, \ldots, u_{d}\right):=1-2 \times 1_{\left\{\left\langle z\left(u_{1}, \ldots, u_{d}\right), u_{1}-u_{0}\right\rangle \leq 0\right\}}, \quad u_{0}, \ldots, u_{d} \in S^{d-1},
$$

where $z\left(u_{1}, \ldots, u_{d}\right):=0$ for points $u_{1}, \ldots, u_{d}$ that are not in general position. To interpret this function we consider the Voronoi tessellation generated by points $u_{0}, \ldots, u_{d} \in S^{d-1}$ in general position. We then have $J\left(u_{0}, \ldots, u_{d}\right)=1$ if and only if $z\left(u_{0}, \ldots, u_{d}\right)$ is pointing along the edge generated by $u_{1}, \ldots, u_{d}$ and starting at the point 0 . We define a probability measure on the interval $[-1,1]$ by

$$
\mathbb{W}:=c_{0}^{-1} \int \cdots \int 1_{\left\{J\left(u_{0}, \ldots, u_{d}\right)\left|z\left(u_{1}, \ldots, u_{d}\right)\right| \in \cdot\right\}} \Delta_{d}\left(u_{0}, \ldots, u_{d}\right) \mathbb{S}\left(\mathrm{d} u_{0}\right) \cdots \mathbb{S}\left(\mathrm{d} u_{d}\right),
$$

where $c_{0}$ is as defined in Theorem 1.1(v) and $\mathbb{S}$ is the uniform distribution on the unit sphere $S^{d-1}$. Note that $\mathbb{W}(\{-1\})=\mathbb{W}(\{1\})=0$. In view of Theorem 1.1(v) and Corollary 2.1, we can interpret $\mathbb{W}$ as the conditional distribution of the signed distance of the typical vertex 
from the centre of a randomly chosen edge emanating from the vertex, given that the distance from the typical vertex to its neighbours is 1 . Another interpretation of $\mathbb{W}$ and a more explicit formula will be given in Proposition 5.2.

The following proposition generalizes a result of [8] and provides the joint distribution of the typical edge and its neighbours. The result can be considered a preliminary one: a more explicit version will be given in Theorem 5.1. As above, it is convenient to introduce the shorthand notation $\left(L^{\prime}, L^{\prime \prime}, \rho_{1}, \Phi_{1}\right) \equiv\left(L^{\prime}(0), L^{\prime \prime}(0), \rho_{1}(0), \Phi_{1}(0)\right)$, where we recall from Section 4 that $\Phi_{1}(x)$ is the set of the normalized directions to the $d$ neighbours of the edge with centre $x \in N_{1}$.

Proposition 5.1. The following assertions hold under $\mathrm{P}_{N_{1}}^{0}$.

(i) The random variables $\left(L^{\prime}, L^{\prime \prime}, \rho_{1}\right)$ and $\Phi_{1}$ are independent.

(ii) The distribution of $\Phi_{1}$ is $\mathbb{Q}_{1}\left(\cdot \times S^{d-1}\right)$, where $\mathbb{Q}_{1}$ is as given in (1.3) for $k=1$.

(iii) For any measurable mappings $h_{1}: \mathbb{R}^{2} \rightarrow[0, \infty)$ and $h_{2}:[0, \infty) \rightarrow[0, \infty)$,

$$
\begin{aligned}
\mathrm{E}_{N_{1}}^{0}\left[h_{1}\left(L^{\prime}, L^{\prime \prime}\right) h_{2}\left(\rho_{1}\right)\right] & \\
=\frac{d \lambda\left(\lambda \kappa_{d}\right)^{d}}{2 \Gamma(d)} \int_{-1}^{1} \int_{0}^{\infty} \int_{0}^{\infty} & \left(h_{1}(r w-t, r w)+h_{1}(-r w,-r w+t)\right) \\
& \times h_{2}\left(r \sqrt{1-w^{2}}\right) V^{*}(t, r, w) \\
& \times \exp \left[-\lambda V\left(t, r, \sqrt{t^{2}+r^{2}-2 t r w}\right)\right] \\
& \times r^{d^{2}-1} \mathrm{~d} t \mathrm{~d} r \mathbb{W}(\mathrm{d} w),
\end{aligned}
$$

where $V^{*}(t, r, w)$ is as defined in (5.1) and $\mathbb{W}$ is as given in (5.2).

Proof. Using Proposition 4.2 instead of Proposition 4.1, the first two assertions can be proved as were the equivalent assertions of Theorem 4.1. To determine the distribution of $\left(L^{\prime}, L^{\prime \prime}, \rho_{1}\right)$ under $\mathrm{P}_{N_{1}}^{0}$, we will now use Corollary 2.1 and an idea that goes back to [4] (see also [8]). Assume that $x_{0}, \ldots, x_{d} \in \mathbb{R}^{d}$ are in general position. Then, for any $i \in\{0, \ldots, d\}$, define

$$
z_{i}\left(x_{0}, \ldots, x_{d}\right):=z\left(x_{0}, \ldots, x_{i-1}, x_{i+1}, \ldots, x_{d}\right)
$$

and note that

$$
z_{i}\left(a x_{0}, \ldots, a x_{d}\right)=a z_{i}\left(x_{0}, \ldots, x_{d}\right), \quad a \neq 0 .
$$

For points $x_{0}, \ldots, x_{d} \in \mathbb{R}^{d}$ that are not in general position, we define $z_{i}\left(x_{0}, \ldots, x_{d}\right):=0$. For a measurable function $h: N \rightarrow[0, \infty)$, using Corollary 2.1 we obtain

$$
2 \lambda_{1} \mathrm{E}_{N_{1}}^{0}[h]=\lambda_{0} \sum_{i=0}^{d} \mathrm{E}_{N_{0}}^{0}\left[h\left(N-R z_{i}\left(U_{0}, \ldots, U_{d}\right)\right)\right],
$$

where $R \equiv R_{0}$ and $\left(U_{0}, \ldots, U_{d}\right) \equiv\left(U_{0,0}, \ldots, U_{0, d}\right)$. 
Next we introduce some random variables as seen from the typical vertex. From $i \in$ $\{0, \ldots, d\}$, we define

$$
\begin{gathered}
J_{i}:=J\left(U_{i}, U_{0}, \ldots, U_{i-1}, U_{i+1}, \ldots, U_{d}\right), \\
Z_{i}:=z_{i}\left(U_{0}, \ldots, U_{d}\right), \quad \eta_{i}:=\left|Z_{i}\right|^{-1} Z_{i}, \\
L_{i}:=L^{\prime \prime}\left(N-R Z_{i}, 0\right)-L^{\prime}\left(N-R Z_{i}, 0\right)=L^{\prime \prime}\left(N, R Z_{i}\right)-L^{\prime}\left(N, R Z_{i}\right) .
\end{gathered}
$$

We also define $U_{(i)}:=U_{i+1}$ for $i \leq d-1$ and $U_{(d)}:=U_{0}$. Let $t>0$. A crucial fact is the equivalence

$$
L_{i}>t \quad \Longleftrightarrow \quad N \cap\left[B\left(t J_{i} \eta_{i},\left|R U_{(i)}-t J_{i} \eta_{i}\right|\right) \backslash B(0, R)\right]=\varnothing .
$$

Assertions (i) and (iii) of Theorem 1.1 imply that

$$
\mathrm{P}_{N_{0}}^{0}\left(L_{i}>t \mid \Psi_{0}, R\right)=\exp \left[-\lambda V\left(t, R, \sqrt{R^{2}+t^{2}-2 t R J_{i}\left\langle U_{(i)}, \eta_{i}\right\rangle}\right)\right] \mathrm{e}^{\lambda \kappa_{d} R^{d}},
$$

i.e.

$$
\mathrm{P}_{N_{0}}^{0}\left(L_{i} \in \mathrm{d} t \mid \Psi_{0}, R\right)=F\left(J_{i}\left\langle U_{(i)}, \eta_{i}\right\rangle, t, R\right) \mathrm{d} t,
$$

where

$$
\begin{aligned}
F(w, t, r) & :=-\mathrm{e}^{\lambda \kappa_{d} r^{d}} \frac{\mathrm{d}}{\mathrm{d} t} \exp \left[-\lambda V\left(t, r, \sqrt{t^{2}+r^{2}-2 t r w}\right)\right] \\
& =\lambda \mathrm{e}^{\lambda \kappa_{d} r^{d}} V^{*}(t, r, w) \exp \left[-\lambda V\left(t, r, \sqrt{t^{2}+r^{2}-2 t r w}\right)\right]
\end{aligned}
$$

Under $\mathrm{P}_{N_{1}}^{0}$ we consider the set, $E:=\left\{L^{\prime} U(0), L^{\prime \prime} U(0)\right\}$, of vertices of the typical edge. From (5.4) and (5.5), for any nonnegative, measurable function $g$ with suitable domain we then obtain

$$
\begin{aligned}
& 2 \lambda_{1} \mathrm{E}_{N_{1}}^{0}\left[g\left(E, \rho_{1}\right)\right] \\
& \quad=\lambda_{0} \sum_{i=0}^{d} \mathrm{E}_{N_{0}}^{0}\left[g\left(\left\{-R Z_{i},-R Z_{i}+L_{i} J_{i} \eta_{i}\right\}, R \sqrt{1-\left|Z_{i}\right|^{2}}\right)\right] \\
& \quad=\lambda_{0} \sum_{i=0}^{d} \mathrm{E}_{N_{0}}^{0}\left[\int g\left(\left\{-R Z_{i},-R Z_{i}+t J_{i} \eta_{i}\right\}, R \sqrt{1-\left|Z_{i}\right|^{2}}\right) F\left(J_{i}\left\langle U_{(i)}, \eta_{i}\right\rangle, t, R\right) \mathrm{d} t\right] .
\end{aligned}
$$

It is now easy to check that

$$
\left\langle U_{(i)}, z_{i}\left(U_{0}, \ldots, U_{d}\right)\right\rangle=\left|z_{i}\left(U_{0}, \ldots, U_{d}\right)\right|^{2}=\left|Z_{i}\right|^{2}, \quad i=0, \ldots, d .
$$

Hence, we have $\left\langle U_{(i)}, \eta_{i}\right\rangle=\left|Z_{i}\right|$. Letting $\xi_{i}:=J_{i}\left|Z_{i}\right|$, and noting that $Z_{i}=\left|Z_{i}\right| \eta_{i}=J_{i} \xi_{i} \eta_{i}$, we can rewrite our previous result as

$$
\begin{aligned}
2 \lambda_{1} \mathrm{E}_{N_{1}}^{0}\left[g\left(E, \rho_{1}\right)\right] & \\
& =\lambda_{0} \sum_{i=0}^{d} \mathrm{E}_{N_{0}}^{0}\left[\int g\left(\left\{-R \xi_{i} J_{i} \eta_{i},-R \xi_{i} J_{i} \eta_{i}+t J_{i} \eta_{i}\right\}, R \sqrt{1-\xi_{i}^{2}}\right) F\left(\xi_{i}, t, R\right) \mathrm{d} t\right] .
\end{aligned}
$$


Let $A_{i}$ denote the event that $-J_{i} Z_{i}<J_{i} Z_{i}$, and let $A_{i}^{\mathrm{c}}$ be its complement. By definition of $\left(L^{\prime}, L^{\prime \prime}\right)$, for any measurable function $h: \mathbb{R}^{2} \times[0, \infty) \rightarrow[0, \infty)$ we obtain

$$
\begin{aligned}
2 \lambda_{1} \mathrm{E}_{N_{1}}^{0} & {\left[h\left(L^{\prime}, L^{\prime \prime}, \rho_{1}\right)\right] } \\
= & \lambda_{0} \sum_{i=0}^{d} \mathrm{E}_{N_{0}}^{0}\left[1_{A_{i}} \int h\left(-R \xi_{i},-R \xi_{i}+t, R \sqrt{1-\xi_{i}^{2}}\right) F\left(\xi_{i}, t, R\right) \mathrm{d} t\right] \\
& +\lambda_{0} \sum_{i=0}^{d} \mathrm{E}_{N_{0}}^{0}\left[1_{A_{i}^{\mathrm{c}}} \int h\left(R \xi_{i}-t, R \xi_{i}, R \sqrt{1-\xi_{i}^{2}}\right) F\left(\xi_{i}, t, R\right) \mathrm{d} t\right] .
\end{aligned}
$$

To conclude the proof of assertion (iii), we apply a symmetry argument based on $\mathbb{Q}_{0}$, the distribution of $\left\{U_{0}, \ldots, U_{d}\right\}$ (see (1.3)) and the independence of $\left(U_{0}, \ldots, U_{d}\right)$ and $R$. For all $u_{0}, \ldots, u_{d} \in S^{d-1}$, we have

$$
z_{i}\left(-u_{0}, \ldots,-u_{d}\right)=-z_{i}\left(u_{0}, \ldots, u_{d}\right)
$$

and, hence,

$$
J_{i}\left(-u_{0}, \ldots,-u_{d}\right)=J_{i}\left(u_{0}, \ldots, u_{d}\right) .
$$

Furthermore, we have $\Delta_{d}\left(-u_{0}, \ldots,-u_{d}\right)=\Delta_{d}\left(u_{0}, \ldots, u_{d}\right)$. It follows that

$$
\begin{aligned}
\sum_{i=0}^{d} \mathrm{E}_{N_{0}}^{0}\left[1_{A_{i}} \int h\left(-R \xi_{i},-R \xi_{i}+t, R \sqrt{1-\xi_{i}^{2}}\right) F\left(\xi_{i}, t, R\right) \mathrm{d} t\right] \\
\quad=\sum_{i=0}^{d} \mathrm{E}_{N_{0}}^{0}\left[1_{A_{i}^{\mathrm{c}}} \int h\left(-R \xi_{i},-R \xi_{i}+t, R \sqrt{1-\xi_{i}^{2}}\right) F\left(\xi_{i}, t, R\right) \mathrm{d} t\right] \\
=\frac{1}{2} \sum_{i=0}^{d} \mathrm{E}_{N_{0}}^{0}\left[\int h\left(-R \xi_{i},-R \xi_{i}+t, R \sqrt{1-\xi_{i}^{2}}\right) F\left(\xi_{i}, t, R\right) \mathrm{d} t\right]
\end{aligned}
$$

This and a similar formula for the second summand on the right-hand side of (5.6) yields

$$
\begin{aligned}
2 \lambda_{1} \mathrm{E}_{N_{1}}^{0} & {\left[h\left(L^{\prime}, L^{\prime \prime}, \rho_{1}\right)\right] } \\
= & \frac{\lambda_{0}}{2} \sum_{i=0}^{d} \mathrm{E}_{N_{0}}^{0}\left[\int h\left(-R \xi_{i},-R \xi_{i}+t, R \sqrt{1-\xi_{i}^{2}}\right) F\left(\xi_{i}, t, R\right) \mathrm{d} t\right] \\
& +\frac{\lambda_{0}}{2} \sum_{i=0}^{d} \mathrm{E}_{N_{0}}^{0}\left[\int h\left(R \xi_{i}-t, R \xi_{i}, R \sqrt{1-\xi_{i}^{2}}\right) F\left(\xi_{i}, t, R\right) \mathrm{d} t\right] .
\end{aligned}
$$

By definition of $\mathbb{W}$ (see (5.2)) and Theorem 1.1(v), we have

$$
\mathbb{W}=\frac{1}{d+1} \sum_{i=0}^{d} \mathrm{P}_{N_{0}}^{0}\left(\xi_{i} \in \cdot\right)
$$


Again, using the independence of $R$ and $\left(\xi_{0}, \ldots, \xi_{d}\right)$, this implies that

$$
\begin{aligned}
2 \lambda_{1} \mathrm{E}_{N_{1}}^{0}\left[h\left(L^{\prime}, L^{\prime \prime}, \rho_{1}\right)\right] & \\
= & \frac{(d+1) \lambda_{0}}{2} \mathrm{E}_{N_{0}}^{0}\left[\iint h\left(-R w,-R w+t, R \sqrt{1-w^{2}}\right) F(w, t, R) \mathrm{d} t \mathbb{W}(\mathrm{d} w)\right] \\
& +\frac{(d+1) \lambda_{0}}{2} \mathrm{E}_{N_{0}}^{0}\left[\iint h\left(R w-t, R w, R \sqrt{1-w^{2}}\right) F(w, t, R) \mathrm{d} t \mathbb{W}(\mathrm{d} w)\right] .
\end{aligned}
$$

Recalling that $2 \lambda_{1}=(d+1) \lambda_{0}$ and that $R^{d}$ has a gamma distribution with shape parameter $d$ and scale parameter $\lambda \kappa_{d}$, we obtain assertion (iii).

Assertion (iii) of the previous theorem implies the following natural symmetry property:

$$
\mathrm{P}_{N_{1}}^{0}\left(\left(L^{\prime}, L^{\prime \prime}, \rho_{1}\right) \in \cdot\right)=\mathrm{P}_{N_{1}}^{0}\left(\left(-L^{\prime \prime},-L^{\prime}, \rho_{1}\right) \in \cdot\right) .
$$

To derive an alternative to $(5.3)$, we introduce the $[-1,1]$-valued random variables

$$
\eta^{\prime}:=-\frac{L^{\prime}}{\sqrt{L^{\prime 2}+\rho_{1}^{2}}}, \quad \eta^{\prime \prime}:=\frac{L^{\prime \prime}}{\sqrt{L^{\prime \prime 2}+\rho_{1}^{2}}} .
$$

We have $\eta^{\prime}=\cos \alpha^{\prime}$ and $\eta^{\prime \prime}=\cos \alpha^{\prime \prime}$ for some random angles $\alpha^{\prime}, \alpha^{\prime \prime} \in[0, \pi]$. Here $\alpha^{\prime}$ is the angle spanned by $U(0)$ and the vector between the edge $L^{\prime} U(0)$ and one of the neighbours of $C_{1}(0)$, while $\alpha^{\prime \prime}$ is the angle spanned by $-U(0)$ and the vector between the edge $L^{\prime \prime} U(0)$ and one of the neighbours of $C_{1}(0)$ (see Figure 2). Since $\sin \left(\alpha^{\prime}+\alpha^{\prime \prime}\right) \geq 0$, it follows that $q\left(\eta^{\prime}, \eta^{\prime \prime}\right) \geq 0$, where

$$
q(w, v):=v \sqrt{1-w^{2}}+w \sqrt{1-v^{2}}
$$

This inequality also follows more directly from $L^{\prime \prime}-L^{\prime} \geq 0$. Under $\mathrm{P}_{N_{1}}^{0}$ there is (almost surely) a one-to-one correspondence between $\left(L^{\prime}, L^{\prime \prime}, \rho_{1}\right)$ and $\left(\eta^{\prime}, \eta^{\prime \prime}, L\right)$.

The next two propositions can be found in [8]. For any $s \in \mathbb{R}$, we denote by $[s]$ the largest integer $k$ such that $k \leq s$.

Proposition 5.2. We have

$$
\mathrm{P}_{N_{1}}^{0}\left(\eta^{\prime} \in \cdot\right)=\mathrm{P}_{N_{1}}^{0}\left(\eta^{\prime \prime} \in \cdot\right)=\mathbb{W} .
$$

Moreover, the measure $\mathbb{W}$ is given by

$$
\mathbb{W}(\mathrm{d} w)=c^{*}\left(1-w^{2}\right)^{\left(d^{2}-d-2\right) / 2} b(w) \mathrm{d} w,
$$

where

$$
c^{*}=\frac{\left(d^{2}-d\right) \Gamma((d+1) / 2) \Gamma\left(d^{2} / 2\right)}{\Gamma(d / 2) \Gamma\left(\left(d^{2}+1\right) / 2\right)}, \quad b(w):=\sum_{i=0}^{[(d-1) / 2]} b_{i}(w) .
$$

The $b_{i}(w)$ are given by

$$
b_{0}(w):= \begin{cases}\pi^{-1}\left((\pi-\arccos (w)) w+\sqrt{1-w^{2}}\right) & \text { if d is even } \\ (1+w) / 2 & \text { if } d \text { is odd }\end{cases}
$$


and, for $i \in\{1, \ldots,[(d-1) / 2]\}$, by

$$
b_{i}(w):= \begin{cases}-\frac{1}{4 \sqrt{\pi}} \frac{\Gamma(i)}{\Gamma\left(i+\frac{3}{2}\right)}\left(1-w^{2}\right)^{i+1 / 2} & \text { if d is even, } \\ -\frac{1}{4 \sqrt{\pi}} \frac{\Gamma\left(i-\frac{1}{2}\right)}{\Gamma(i+1)}\left(1-w^{2}\right)^{i} & \text { if } d \text { is odd } .\end{cases}
$$

Proof. Here we prove only the first assertion. The second will be derived in the proof of Proposition 5.3. We choose $\omega, \omega^{\prime} \in N$ such that $0 \in N_{0}(\omega)$ and $\omega^{\prime}=\omega-x$ for some (unique) $x \in N_{1}(\omega)$ satisfying $0 \in C_{1}(\omega, x)$. We then define $\alpha\left(\omega, \omega^{\prime}\right) \in[0, \pi]$ as the angle spanned by the vector between 0 and one of the neighbours of $C_{1}(\omega, x)$ and the (directed) edge $C_{1}(\omega, x)$ starting at 0 . For all other $\omega, \omega^{\prime} \in N$, we give $\alpha\left(\omega, \omega^{\prime}\right)$ some fixed value in $[0, \pi]$. For $j=0$ and $k=1$, Proposition 2.2 yields, for any measurable function $f:[-1,1] \rightarrow[0, \infty)$,

$$
\lambda_{0} \mathrm{E}_{N_{0}}^{0}\left[\sum_{x \in N_{1}, 0 \in C_{1}(x)} f(\cos \alpha(N, N-x))\right]=\lambda_{1} \mathrm{E}_{N_{1}}^{0}\left[\sum_{x \in N_{0}, x \in C_{1}(0)} f(\cos \alpha(N-x, N))\right] .
$$

Using the notation established in the proof of Proposition 5.1, the left-hand side of this equation can be written as

$$
\lambda_{0} \mathrm{E}_{N_{0}}^{0}\left[\sum_{i=0}^{d} f\left(\xi_{i}\right)\right]=(d+1) \lambda_{0} \int f(w) \mathbb{W}(\mathrm{d} w) ;
$$

see (5.7). However, the right-hand side of (5.13) equals

$$
\lambda_{1} \mathrm{E}_{N_{1}}^{0}\left[f\left(\eta^{\prime}\right)+f\left(\eta^{\prime \prime}\right)\right] .
$$

Now, (5.8) implies that $\eta^{\prime}$ and $\eta^{\prime \prime}$ have the same distribution under $\mathrm{P}_{N_{1}}^{0}$. Therefore,

$$
(d+1) \lambda_{0} \int f(w) \mathbb{W}(\mathrm{d} w)=2 \lambda_{1} \mathrm{E}_{N_{1}}^{0}\left[f\left(\eta^{\prime}\right)\right] .
$$

As $(d+1) \lambda_{0}=2 \lambda_{1}$, the assertion in (5.10) follows.

We define $L:=L^{\prime \prime}-L^{\prime}$ as the length of the typical edge.

Proposition 5.3. The joint distribution of $\eta^{\prime}, \eta^{\prime \prime}$, and $L$ under $\mathrm{P}_{N_{1}}^{0}$ is given by

$$
\begin{aligned}
& \mathrm{P}_{N_{1}}^{0}\left(\left(\eta^{\prime}, \eta^{\prime \prime}, L\right) \in \cdot\right) \\
& =\frac{d^{2}\left(\lambda \kappa_{d}\right)^{d+1}}{\Gamma(d)} c^{*} \int_{0}^{\infty} \int_{-1}^{1} \int_{-1}^{1} 1_{\{(w, v, t) \in \cdot\}} 1_{\{q(w, v)>0\}} b(w) b(v) \\
& \times \frac{\left(1-w^{2}\right)^{\left(d^{2}-2\right) / 2}\left(1-v^{2}\right)^{\left(d^{2}-2\right) / 2}}{q(w, v)^{d^{2}+d}} t^{d^{2}+d-1} \\
& \times \exp \left[-\lambda t^{d} V\left(1, \frac{\sqrt{1-v^{2}}}{q(w, v)}, \frac{\sqrt{1-w^{2}}}{q(w, v)}\right)\right] \mathrm{d} w \mathrm{~d} v \mathrm{~d} t
\end{aligned}
$$

where $c^{*}, b(w)$, and $q(w, v)$ are as given in (5.12) and (5.9), respectively. 
Proof. As

$$
\exp \left[-\lambda V\left(t, r, \sqrt{t^{2}+r^{2}-2 t r w}\right)\right] \rightarrow 0 \quad \text { as } t \rightarrow \infty
$$

and $\left[d\left(\lambda \kappa_{d}\right)^{d} / \Gamma(d)\right] r^{d^{2}-1} \mathrm{e}^{-\lambda \kappa_{d} r^{d}}$ is a density, for all $w \in[-1,1]$ we obtain

$$
\frac{d \lambda\left(\lambda \kappa_{d}\right)^{d}}{\Gamma(d)} \int_{0}^{\infty} \int_{0}^{\infty} V^{*}(t, r, w) \exp \left[-\lambda V\left(t, r, \sqrt{t^{2}+r^{2}-2 t r w}\right)\right] r^{d^{2}-1} \mathrm{~d} t \mathrm{~d} r=1 .
$$

For measurable mappings $h_{1}:[-1,1]^{2} \rightarrow[0, \infty)$ and $h_{2}:[0, \infty) \rightarrow[0, \infty)$, from $(5.3)$ we obtain

$$
\begin{aligned}
\mathrm{E}_{N_{1}}^{0}\left[h_{1}\left(\eta^{\prime}, \eta^{\prime \prime}\right) h_{2}(L)\right] & \\
=\frac{d \lambda\left(\lambda \kappa_{d}\right)^{d}}{2 \Gamma(d)} \iiint & \left(h_{1}\left(\frac{t-r w}{\sqrt{r^{2}+t^{2}-2 t r w}}, w\right)+h_{1}\left(w, \frac{-r w+t}{\sqrt{r^{2}+t^{2}-2 t r w}}\right)\right) \\
& \times h_{2}(t) V^{*}(t, r, w) \exp \left[-\lambda V\left(t, r, \sqrt{\left.t^{2}+r^{2}-2 t r w\right)}\right)\right] \\
& \times r^{d^{2}-1} \mathrm{~d} r \mathbb{W}(\mathrm{d} w) \mathrm{d} t .
\end{aligned}
$$

To modify the inner integration we write

$$
r(w, t, v):=\frac{t \sqrt{1-v^{2}}}{q(w, v)}, \quad r \geq 0, w, v \in[-1,1],
$$

provided that $q(w, v)>0$. It is easy to check that, for any fixed $t>0$ and $w \in(-1,1)$, $v \mapsto r(w, t, v)$ is a differentiable bijection between $(0, \infty)$ and $\{v \in(-1,1): q(w, v)>0\}$. Moreover, we have

$$
\begin{gathered}
\frac{\partial}{\partial v} r(w, t, v)=-\frac{t \sqrt{1-w^{2}}}{q(w, v)^{2} \sqrt{1-v^{2}}}, \\
t-r(w, t, v) w=\frac{t v \sqrt{1-w^{2}}}{q(w, v)} \\
t^{2}+r(w, t, v)^{2}-2 r(w, t, v) w t=\frac{t^{2}\left(1-w^{2}\right)}{q(w, v)^{2}} .
\end{gathered}
$$

Substituting these equations into (5.16) yields

$$
\mathrm{E}_{N_{1}}^{0}\left[h_{1}\left(\eta^{\prime}, \eta^{\prime \prime}\right) h_{2}(L)\right]=\iiint\left(h_{1}(v, w)+h_{1}(w, v)\right) h_{2}(t) H(w, t, v) \mathrm{d} v \mathrm{~d} t \mathbb{W}(\mathrm{d} w),
$$

where

$$
\begin{aligned}
H(w, t, v):= & \frac{d \lambda\left(\lambda \kappa_{d}\right)^{d}}{2 \Gamma(d)} 1_{\{q(w, v)>0\}} \frac{t \sqrt{1-w^{2}}}{q(w, v)^{2} \sqrt{1-v^{2}}} V^{*}\left(t, \frac{t \sqrt{1-v^{2}}}{q(w, v)}, w\right) \\
& \times\left(\frac{t \sqrt{1-v^{2}}}{q(w, v)}\right)^{d^{2}-1} \exp \left[-\lambda V\left(t, \frac{t \sqrt{1-v^{2}}}{q(w, v)}, \frac{t \sqrt{1-w^{2}}}{q(w, v)}\right)\right] .
\end{aligned}
$$


In particular,

$$
\mathrm{E}_{N_{1}}^{0}\left[h_{1}\left(\eta^{\prime}, \eta^{\prime \prime}\right)\right]=\iint\left(h_{1}(v, w)+h_{1}(w, v)\right) H^{*}(w, v) \mathrm{d} v \mathbb{W}(\mathrm{d} w)
$$

where

$$
H^{*}(w, v):=\int_{0}^{\infty} H(w, t, v) \mathrm{d} t .
$$

Equation (5.15) implies that

$$
\int_{-1}^{1} H^{*}(w, v) \mathrm{d} v=\frac{1}{2}, \quad w \in[-1,1] .
$$

Hence, it follows from (5.19) that

$$
\mathrm{P}\left(\eta^{\prime} \in \cdot\right)=\frac{1}{2} \mathbb{W}+\iint 1_{\{v \in \cdot\}} H^{*}(w, v) \mathrm{d} v \mathbb{W}(\mathrm{d} w) .
$$

Using (5.10), we obtain the invariance relationship

$$
\mathbb{W}=\int_{-1}^{1} \int_{-1}^{1} 1_{\{v \in \cdot\}} 2 H^{*}(w, v) \mathrm{d} v \mathbb{W}(\mathrm{d} w)
$$

We next compute the kernel function $H^{*}(w, v)$. From [8, Equation (11)],

$$
V^{*}\left(t, \frac{t \sqrt{1-v^{2}}}{q(w, v)}, w\right)=d \kappa_{d} t^{d-1}\left(\frac{\sqrt{1-w^{2}}}{q(w, v)}\right)^{d-1} b(v),
$$

where $b(v)$ is as given in (5.12). Recalling definition (5.18), we hence obtain

$$
2 H(w, t, v)=S(w, t, v)\left(1-v^{2}\right)^{\left(d^{2}-d-2\right) / 2} b(v),
$$

where

$$
\begin{aligned}
S(w, t, v):= & 1_{\{q(w, v)>0\}} \frac{d^{2}\left(\lambda \kappa_{d}\right)^{d+1}}{\Gamma(d)} \frac{\left(1-w^{2}\right)^{d / 2}\left(1-v^{2}\right)^{d / 2}}{q(w, v)^{d^{2}+d}} t^{d^{2}+d-1} \\
& \times \exp \left[-\lambda V\left(t, \frac{t \sqrt{1-v^{2}}}{q(w, v)}, \frac{t \sqrt{1-w^{2}}}{q(w, v)}\right)\right] .
\end{aligned}
$$

As $V(t, t a, t b)=t^{d} V(1, a, b)$ for all $a, b, t \geq 0$, we find from an easy calculation that

$$
\int_{0}^{\infty} S(w, t, v) \mathrm{d} t=1_{\{q(w, v)>0\}} d^{2} \kappa_{d}^{d+1} \frac{\left(1-w^{2}\right)^{d / 2}\left(1-v^{2}\right)^{d / 2}}{q(w, v)^{d^{2}+d}} G(w, v)^{-d-1},
$$

where

$$
G(w, v):=V\left(1, \frac{\sqrt{1-v^{2}}}{q(w, v)}, \frac{\sqrt{1-w^{2}}}{q(w, v)}\right) .
$$

This is a symmetric function of $w$ and $v$, and it immediately follows that the right-hand side of (5.11) does satisfy (5.20). Moreover, $H^{*}(w, v)$ is continuous and positive on $\{(w, v) \in$ $\left.(-1,1)^{2}: q(w, v)>0\right\}$, from which (5.11) follows since standard results on discrete-time 
Markov processes imply that there exists at most one probability measure $\mathbb{W}$ on $[-1,1]$ satisfying both (5.20) and $\mathbb{W}(\{-1\})=\mathbb{W}(\{1\})=0$. Indeed, we may consider $2 H^{*}(w, v) \mathrm{d} v$ a stochastic kernel on $(-1,1)$. The associated Markov process has the regeneration set $[-1 / 2,1 / 2]$, for instance. By Theorem VII.3.5 of [1], the process can have at most one invariant measure.

The value (see (5.12)) of the constant $c^{*}$ in (5.11) is a consequence of [8, Equation (13)]. Finally, we may substitute (5.21), (5.22), and (5.11) into (5.17) to obtain (5.14).

We can now summarize the main results of this section, as follows.

Theorem 5.1. Under $\mathrm{P}_{N_{1}}^{0}$ the following assertions hold.

(i) The random variables $\left(\eta^{\prime}, \eta^{\prime \prime}\right), G\left(\eta^{\prime}, \eta^{\prime \prime}\right) L^{d}$, and $\Phi_{1}$ are independent.

(ii) The random variable $G\left(\eta^{\prime}, \eta^{\prime \prime}\right) L^{d}$ has a gamma distribution with shape parameter $d+1$ and scale parameter $\lambda$.

(iii) The distribution of $\left(\eta^{\prime}, \eta^{\prime \prime}\right)$ has the density

$$
d^{2} \kappa_{d}^{d+1} c^{*} 1_{\{q(w, v)>0\}} b(w) b(v) \frac{\left(1-w^{2}\right)^{\left(d^{2}-2\right) / 2}\left(1-v^{2}\right)^{\left(d^{2}-2\right) / 2}}{q(w, v)^{d^{2}+d}} G(w, v)^{-d-1} .
$$

(iv) The distribution of $\Phi_{1}$ is $\mathbb{Q}_{1}\left(\cdot \times S^{d-1}\right)$, where $\mathbb{Q}_{1}$ is as given in (1.3) for $k=1$.

Proof. Assertion (iv) follows directly from Proposition 5.1 while assertions (ii) and (iii) follow from (5.14) and a simple substitution. Combining Proposition 5.1 and (5.14) yields assertion (i).

Remark 5.1. Under $\mathrm{P}_{N_{1}}^{0}$ the origin is almost surely the centre of the typical edge. The random variable $G\left(\eta^{\prime}, \eta^{\prime \prime}\right) L^{d}$ is the volume of the union of the two balls, respectively centred at the endpoints of the typical edge, whose radii are given by the respective distances from the endpoints to one of the neighbours of the edge. This volume, the angles $\arccos \eta^{\prime}$ and $\arccos \eta^{\prime \prime}$, and the normalized directions in $\Phi_{1}$ provide a complete geometric description of the typical edge and its neighbours. Theorem 5.1 gives remarkably explicit formulae for the distribution of these random variables. With a little more effort, Theorem 5.1 can be deduced from Propositions 4.2 and 2.1 .

\section{Acknowledgements}

We thank two anonymous referees for their careful and critical reading of our paper. The research of the second author was supported in part by a Marie Curie Transfer of Knowledge Fellowship of the European Union under contract number MTDK-CT-2004-013389.

\section{References}

[1] Asmussen, S. (2003). Applied Probability and Queues, 2nd edn. Springer, New York.

[2] Kallenberg, O. (2002). Foundations of Modern Probability, 2nd edn. Springer, New York.

[3] Mecke, J. (1967). Stationäre zufällige Maße auf lokalkompakten Abelschen Gruppen. Z. Wahrscheinlichkeitsth. 9, 36-58.

[4] Mecke, J. And Muche, L. (1995). The Poisson Voronoi tessellation. I. A basic identity. Math. Nachr. 176, 199-208.

[5] Miles, R. (1974). A synopsis of 'Poisson flats in Euclidean spaces'. In Stochastic Geometry, eds E. F. Harding and D. G. Kendall, John Wiley, New York, pp. 202-227.

[6] MøLler, J. (1989). Random tessellations in $\mathbb{R}^{d}$. Adv. Appl. Prob. 21, 37-73. 
[7] Møller, J. (1994). Lectures on Random Voronoi Tessellations (Lecture Notes Statist. 87). Springer, New York.

[8] Muche, L. (2005). The Poisson-Voronoi tessellation: relationships for edges. Adv. Appl. Prob. 37, $279-296$.

[9] Neveu, J. (1977). Processus ponctuels. In École d'Eté de Probabilités de Saint-Flour VI (Lecture Notes Math. 598). Springer, Berlin, pp. 249-445.

[10] SchneIder, R. AND WeIL, W. (2000). Stochastische Geometrie. Teubner, Stuttgart.

[11] Stoyan, D., Kendall, W. S. And Mecke, J. (1995). Stochastic Geometry and Its Applications, 2nd edn. John Wiley, Chichester. 